\title{
REVISIÓN DE LOS DETERMINANTES MACROECONÓMICOS DEL CONSUMO TOTAL DE LOS HOGARES PARA EL CASO COLOMBIANO
}

\author{
Juan Nicolás Hernández A*
}

\begin{abstract}
Resumen
A la luz de la historia comprendida en el periodo 1954-2002, que abarca episodios de creciente volatilidad en el producto para Colombia, el objetivo del trabajo es hallar empíricamente a partir de la información macroeconómica disponible y en el contexto de las diversas teorías, que variables guardan una relación de largo plazo con el consumo de los hogares y cuantificar su incidencia. Adicionalmente y partiendo de las especificaciones encontradas determinar la calidad del pronóstico, evaluando la posible contribución de su bondad a la discusión teórica. Dentro de los resultados se corrobora para el caso colombiano que la estructura demográfica no resulta tan relevante, el efecto negativo de la tasa de interés, el papel de la riqueza y el ingreso disponible, así como el rol del sistema financiero. Hacia el largo plazo los hogares con un mayor acceso al sistema financiero tenderían a ahorrar.
\end{abstract}

Clasificación JEL: E21, E27, C32

\section{MOTIVACIÓN}

En la medida que para muchos países el consumo representa entre un 50\% a un $70 \%$ del producto $^{1}$ no es sorprendente que la función consumo sea una de las relaciones agregadas de gasto más estudiadas. De hecho la multiplicidad de enfoques teóricos relacionados, sobre los cuales se hará un breve recuento, así lo confirman.

Si bien el tema del consumo no es un tema nuevo, tampoco es un tema culminado. Tras la revisión de la literatura y la observación \& ciertos casos en particular (comentados más adelante) queda claro que los determinantes del consumo no son inmutables y en cierta forma, si bien existen relaciones casi evidentes con variables como el ingreso, la edad entre otras, la obviedad de las mismas en el plano empírico no resulta tan contundente.

\footnotetext{
* Agradezco los valiosos comentarios y sugerencias de Hernando Vargas, Marc Hofstetter, Orlando Gracia, Martha Misas, Juan Mauricio Ramírez, Christian Jaramillo, Lina Cardona y Mario Nigrinis.

1 En particular para Colombia durante la década 19942004 el consumo de los hogares representa en promedio el $63.5 \%$ del PIB
} 
Las múltiples respuestas que surgen ante la necesidad de entender hacia dónde se dirige el consumo han llevado a que autores como Fernández Corugedo (2004) afirmen que no existe una teoría absoluta que explique el comportamiento de este en todas las economías. Lo pertinente es investigar para cada caso particular y en determinados periodos, qué relaciones avalan la dinámica de la variable dentro de uno u otro enfoque.

El simple hecho de partir de un agente representativo para modelar y verificar la dinámica del consumo agregado se percibe como una solución sumamente simplificada. Para algunos autores como Deaton (1992) este tipo de agente tiene dos fallos: "sabe demasiado y vive demasiado", , resultando poco probable que "un agregado de individuos con vidas finitas y con información limitada se comporte como el individuo de los libros de texto"”3.

En esa línea, el objetivo del trabajo es el de hallar empíricamente, a partir de la información macroeconómica disponible para el caso colombiano y en el contexto de las diversas teorías del consumo, cuáles variables guardan una relación de largo plazo con el consumo de los hogares y cuantificar su incidencia.

Al mismo tiempo, una vez identificada la relación de largo plazo, se determinará para los años más recientes, cuándo resulta posible o no, predecir el consumo a ciertos horizontes partiendo de las relaciones halladas. Esta evaluación más que una curiosidad empírica, constituye un aporte a la discusión teórica en la medida que permite inferir acerca de la pertinencia de las conclusiones derivadas de los diversos enfoques teóricos.

Adicionalmente, h evaluación para el caso colombiano es de especial interés por cuanto el periodo de estudio comprende un episodio reciente de recesión y creciente volatilidad del producto no considerado en estudios precedentes. La evidencia indicará, adicionalmente, la conveniencia de aceptar las implicaciones de política económica de uno u otro enfoque teórico de acuerdo con la dinámica y los determinantes encontrados.

\footnotetext{
${ }^{2}$ Del prefacio del libro Understanding Consumption, Angus Deaton 1992.

${ }^{3}$ Ibid.
} 
En ese orden el documento, tras la presente motivación hace un breve recuento de los principales enfoques teóricos que abordan el problema del consumo, al tiempo que rescata alguna evidencia empírica general que pretende evaluar su compatibilidad con lo hechos. Dentro de esta evidencia se evalúa la validez del acierto o desacierto del pronóstico como elemento relevante dentro de la discusión teórica. La corroboración empírica se amplía para el caso colombiano, contrastando la información disponible y los resultados obtenidos por estudios anteriores con las nuevas estimaciones, de cuya metodología se hace una sucinta alusión. Para los sistemas hallados se evalúa la calidad del pronóstico al tiempo que se ponen en contexto algunos de los hallazgos, para finalmente concluir.

\section{TEORÍA DEL CONSUMO}

Existen tres modelos fundamentales sobre los cuales se sustentan las distintas teorías del consumo. La hipótesis del ingreso absoluto (HIA) de Keynes (1936), la hipótesis del ingreso permanente (HIP) de Friedman (1957) y la teoría del ciclo de vida (HCV) de Modigliani (1949).

El trabajo de Keynes (1936) se constituye para muchos en el pilar de la investigación macroeconómica de la función consumo y se basa en el sencillo principio según el cual el gasto en consumo esta en función del ingreso disponible. Solo un incremento en el ingreso agregado, después de impuestos, lleva a un aumento del consumo.

Las medidas de política económica influyen indirectamente sobre el consumo al poder afectar el ingreso disponible. El punto relevante de este enfoque es hacer explícito un aspecto tenido en cuenta en la mayoría de variantes teóricas posteriores, el efecto del ingreso corriente sobre el consumo.

Diversos autores ${ }^{4}$ destacan sin embargo, que especificaciones basadas en el principio de la HIA han resultado insuficientes al pretender proyectar la evolución del consumo. El hecho

\footnotetext{
${ }^{4}$ Véase entre otros Eitrheim O. et al. (2000) y Fernández Corugedo (2004).
} 
de asumir un individuo que observa tan solo su ingreso corriente y omite la intemporalidad en la toma de sus decisiones, restringe la bondad de pronóstico de este tipo de modelos ${ }^{5}$.

La HIP, por su parte, se sustenta en la maximización del bienestar esperado a lo largo de la vida a través de la definición de una función de utilidad. El gasto no se restringe solo por el ingreso del periodo corriente sino por el ingreso esperado. Se discrimina así el ingresoen cada periodo entre permanente y transitorio y de igual forma el consumo. El componente permanente del ingreso reflejaría aquellos factores determinísticos como el capital humano acumulado en tanto que el componente transitorio reflejaría fluctuaciones accidentales.

El consumo permanente presenta una propensión marginal constante con respecto al ingreso permanente, pero al mismo tiempo esta propensión puede variar si alguna de las variables previamente contempladas como dadas (preferencias del consumidor, tasa de interés) se altera.

En presencia de incertidumbre, la HIP exige establecer la manera en que los agentes actualizan sus expectativas de ingreso. Una variante importante de este enfoque teórico es que Friedman(1957) supone expectativas adaptativas. El ingreso permanente se aproxima a partir de la evolución presente e histórica del ingreso. De esta forma si bien los consumidores en la toma de sus decisiones de gasto se preocupan solo por el presente y el futuro, el pasado finalmente incide.

La función consumo en el contexto de la HIP no puede ser formulada en términos de los gastos en consumo y el ingreso disponible. Debe diferenciarse claramente el componente transitorio y permanente de estas variables.

En términos de política habría efectos directos no solo a través de la tasa de interés. El tipo de choques es importante en la percepción y la conducta de los consumidores. Así, si se

\footnotetext{
5 No obstante "Keynes era consciente que cambios inesperados en los valores del capital, cambios sustanciales en la tasa de interés, así como cambios en la distribución del ingreso podían tener efectos significativos sobre la propensión marginal a consumir"
} 
anuncia que una reducción de impuestos o de tasa de interés es permanente el comportamiento es diferente a si la política se percibe como transitoria.

La HCV reconoce también asignaciones de consumo condicionadas a la evolución futura del ingreso, pero adicionalmente advierte variaciones sistemáticas de acuerdo a las necesidades del individuo a lo largo de su vida, es decir, de acuerdo con factores como la edad y los recursos disponibles. Alguna evidencia sugiere sin embargo que restringir los patrones de consumo a un ciclo particular de vida puede deferir de conductas donde el individuo no consume todo su ingreso al final de sus días. El individuo de hecho puede heredar parte de su ingreso acumulado a las generaciones ve nideras ${ }^{6}$.

Las implicaciones de política dadas para el caso del ingreso permanente aplican en el contexto de la $\mathrm{HCV}$, pero adicionalmente la distribución de edades en la economía, la edad de jubilación y la expectativa de vida son importantes en la explicación del consumo.

El punto de las expectativas abre paso a una de las críticas más contundentes que lleva a cuestionar la posibilidad de estimar una función consumo. Lucas (1976) afirma que bajo expectativas racionales (y no adaptativas) la relación estructural entre las variables no existe. De acuerdo a la HIP y la HCV los agentes perciben una relación estructural entre el ingreso permanente y el consumo y, debido a las expectativas adaptativas, existe una relación estructural entre el ingreso permanente y el ingreso observado. Por lo tanto, el consumo es determinado por el ingreso observado.

Si bien Lucas concuerda con la relación entre ingreso permanente y el consumo, no encuentra razón que sustente una relación estable entre el ingreso permanente y el ingreso observado. Ante decisiones de política, los agentes alteran la forma en que infieren el ingreso permanente a partir del observado. Si bien el consumo depende del ingreso futuro este no tiene por que estar relacionado con el pasado. La crítica de Lucas implica la consideración empírica y teórica de las expectativas racionales. Se propone seguir

\footnotetext{
${ }^{6}$ Al respecto véase Bernheim (1985).
} 
modelando un agente que procura maximizar su utilidad a lo largo de la vida pero acentuando el papel de las expectativas y la riqueza.

En esa línea, Hall (1978) propone un modelo de ingreso permanente bajo incertidumbre. En el se pretende maximizar el valor esperado de la función de utilidad sujeta a la evolución de los activos, donde las ganancias derivadas del capital humano son estocásticas y al mismo tiempo la única fuente de incertidumbre. Los agentes deciden su gasto dada toda la información disponible al momento de formar sus expectativas. El ingreso permanente se define como una proporción de la riqueza esperada a lo largo de la vida y a su vez el consumo está en función de dicho ingreso permanente.

Si los consumidores no perciben que ninguna información sobre el futuro se anticipa, su ingreso permanente se mantendría invariante de manera que el consumo mañana seria igual al de hoy. Como la nueva información es impredecible por definición, el consumo presente difiere del de antes solo por un término de perturbación y en consecuencia según la hipótesis del ingreso permanente con expectativas racionales (HIPER) ninguna otra variable, diferente al propio consumo rezagado podría predecir mejor el consumo presente.

Valga resaltar como lo hace Deaton $(1992)^{7}$ que esto no implica que el ingreso no juegue un papel en la determinación del consumo. Ciertamente, el nivel al cual el consumo se fija inicialmente depende de los recursos a lo largo de la vida, determinados al menos en parte por los niveles del ingreso laboral corriente y futuro. Adicionalmente el término de perturbación propio de la caminata aleatoria, refleja cambios inesperados en el ingreso. $\mathrm{Si}$ el ingreso cambia en una forma no prevista, un nuevo patrón de consumo puede generarse de manera que el nivel corriente y futuro del consumo puede cambiar. La proposición de que el consumo sigue una caminata aleatoria no precluye la correlación entre los cambios en el consumo y los cambios en el ingreso corriente.

\footnotetext{
${ }^{7}$ Deaton (1992) Pág. 27
} 
Obsérvese que el modelo de ingreso permanente con expectativas racionales tiene una interesente consecuencia de política económica. Los cambios esperados en el futuro pueden afectar el consumo hoy aún cuando la política no se haya implementado.

En lo que se refiere al impacto de la tasa de interés sobre el consumo, bajo este enfoque teórico, el efecto es ambiguo. La relación positiva entre la variación del interés y el consumo, efecto ingreso, surge como respuesta a unas menores necesidades de ahorro cuando la remuneración del ingreso no consumido aumenta y por ende el individuo destina menos recursos a ahorrar.

Por otra parte la relación negativa entre el consumo y la tasa de interés surge cuando por un lado, ante aumentos en la tasa de interés los activos futuros pierden valor y por ende el individuo se preocupa por ahorrar (efecto riqueza), y por otro cuando el creciente costo de oportunidad del consumo lleva a su disminución (efecto sustitución). De tal manera que el efecto neto de un aumento de tasa de interés pudiera ser positivo o negativo, dependiendo si la suma del efecto sustitución y riqueza logra compensar o no el efecto ingreso.

A pesar de que según la HIPER el consumo se comporta casi como una caminata aleatoria, algunos resultados empíricos podrían refutar este enfoque. Si la predicción del consumo se relaciona con el ingreso pasado, fenómeno reseñado por algunos autores como exceso de sensibilidad (Flavin 1981), cambios en el ingreso corriente pueden tener un impacto significativo y positivo sobre la evolución del consumo de los hogares desafiando el propósito general de la HIPER según la cual el consumo sería menos volátil que el ingreso actual por cuanto el primero se relaciona con el ingreso permanente.

Sobre este último punto, la observación de mercados de capitales imperfectos sustenta en buena parte el efecto del ingreso corriente sobre el consumo. De existir restricciones crediticias, las posibilidades de suavizar el consumo a lo largo del tiempo se limitan y, por 
ende, variaciones en el consumo se relacionan estrechamente con variaciones en el ingreso ${ }^{8}$.

Otro aspecto relacionado con la suavización del consumo tiene que ver con la anotación de Carroll y Summers (1989) quienes, enfatizando la idea original de Friedman, resaltan que el ingreso permanente no debe ser entendido como las ganancias esperadas a lo largo de la vida sino como el ingreso promedio a una edad determinada. Los individuos enfrentan limitaciones en la formación de sus expectativas y si suavizan lo hacen para varios años y no para varias décadas. De esta forma la relación entre el ingreso y el consumo sería más estrecha de lo que predeciría la HIPER.

La suavización del consumo puede depender además de ciertas características particulares al individuo. Por ejemplo Kimball (1990) advierte sobre la importancia del ahorro con motivo precaución. En la medida que el componente estocástico, el ingreso laboral, sea más volátil (tenga una mayor varianza) y dentro de la definición de su función de utilidad el agente incorpore un alto parámetro de prudencia, el nivel de consumo será menor y mayor su ahorro con motivo precaución.

Una versión extendida del ahorro con motivo precaución es el modelo de reservas reguladas que involucra actitudes frente al riesgo. En él los agentes fijan una especie de meta de riqueza que depende negativamente de su nivel de impaciencia y positivamente de la incertidumbre de su ingreso laboral ${ }^{9}$. Estas fuerzas opuestas regulan el proceso de acumulación y en consecuencia las variaciones en el crecimiento del consumo. Carroll (1997) menciona como los consumidores bajo este esquema de reservas reguladas terminan fijando el crecimiento promedio del consumo de acuerdo al crecimiento promedio del ingreso laboral.

Adicionalmente, en línea con la idea original de Dusenberry (1949), quien destaca la relatividad de la utilidad que genera el consumo a lo largo del tiempo, Carroll (2000)

\footnotetext{
${ }^{8}$ Para mayor claridad de las implicaciones de las restricciones crediticias, la representación del Anexo 1 es de utilidad. Se ahonda en el problema por cuanto la evaluación empírica posterior ratifica su relevancia.

${ }^{9}$ Este a su vez relacionado con la posibilidad de quedar desempleado.
} 
menciona como los hábitos generados por el historial del consumo de 1 individuo afectan la relación ingreso-consumo. Si los individuos han logrado un determinado nivel de consumo, puede que sus ahorros con motivo precaución resulten insuficientes para protegerlos no solo de choques negativos en el ingreso, sino para cubrir unos hábitos cada vez más exigentes que terminan por reducir la utilidad futura del consumo.

\section{CORROBORACIÓN EMPÍRICA - HECHOS GENERALES}

Como se anoto previamente, la HIA, al centrarse en el efecto del ingreso disponible corriente sobre el consumo y omitir el papel de las expectativas en la valoración del ingreso, ha llevado a que múltiples estimaciones fundamentadas en dicha hipótesis resulten insuficientes al tratar de caracterizar el comportamiento del consumo. Por ejemplo a mediados de los 80 la desregulación financiera en el Reino Unido y los países escandinavos derivó en una notable disminución en la bondad del pronóstico de las funciones de consumo para entonces estimadas.

El hecho fue interpretado por muchos economistas como un experimento natural que corroboraba las predicciones de las expectativas y el ingreso permanente. De acuerdo a esta interpretación, estimulados por la desregulación financiera, los consumidores revisaron su renta permanente generando un cambio enorme en la correlación entre el consumo y la renta actual, base de la función convencional de consumo.

La corroboración empírica de la hipótesis del ingreso permanente, tampoco está exenta de dificultades. Tal vez la principal esta relacionada con la cuestión de hallar una proxy adecuada del componente permanente, independientemente del tipo de expectativas que se consideren $^{10}$.

En consecuencia, en el caso de la HIP con expectativas adaptativas varios hechos incidieron en su momento para desvirtuar este enfoque como explicación factible del comportamiento del consumo agregado. De forma similar a los desaciertos en la caracterización de la

\footnotetext{
${ }^{10}$ Véase Fernández Corugedo (2004).
} 
evolución del consumo bajo la HIA, Deaton (1992) y Fernández Corugedo (2004) señalan que ante la volatilidad de la economía global hacia la década de los 70, las mediciones propuestas de ingreso permanente a partir del observado subestimaron el consumo. Las relaciones previamente encontradas como estables entre el consumo y el ingreso corriente ${ }^{11}$ no se corroboraron.

Sólo después del trabajo de Davidson (1978) se tuvo conciencia de algunos fallos en la evaluación empírica de los modelos, y en consecuencia sobre el requerimiento de técnicas como el análisis de cointegración y del método de corrección de errores cruciales para la distinción de la dinámica y las relaciones de largo y corto plazo.

Las aplicaciones corroboraron que una relación estacionaria o de equilibrio de largo plazo entre el consumo corriente y el ingreso corriente es improbable si los componentes tendenciales de estas variables exhiben divergencias significativas. Es necesario suponer además que el consumo depende de otras variables "secundarias" adicionales al ingreso de estado estacionario, tales como la riqueza personal, los precios relativos y medidas de distribución de edades e ingreso ${ }^{12}$.

De hecho, especificaciones revisadas de la función consumo que incluyeron mediadas más amplias del bienestar de los hogares, Brodin y Nymoen (1989), (1992) y Berg (1995) (modelos que no parten de condiciones como las de Euler fruto de la lógica propuesta por la modelación que incorpora la HIPER), resultaron exitosas a posteriori en la identificación del cambio que representó la desregulación financiera de mitad de los 80 en los países mencionados.

Al ampliar las variables que pretenden medir el bienestar de los hogares, han resultado determinantes en su relación con los patrones de consumo no solo aquellas asociadas con la riqueza relativa y la estructura de edades. Para casos particulares, como en el Japón,

\footnotetext{
11 Recuérdese que el ingreso corriente incide en la formación del ingreso permanente de acuerdo a las expectativas adaptativas.

${ }^{12} \mathrm{Si}$ el consumo depende de otras variables además del ingreso, una política que altere este último no garantiza un cambio correspondiente en el consumo, a menos que las otras variables sean totalmente insensibles a la política implementada (posibilidad de efectos compensados).
} 
Wakabayashi y Horioka (2005) ${ }^{13}$ muestran como las restricciones al crédito ${ }^{14}$ han incidido en el impacto positivo y significativo del ingreso corriente sobre el consumo en dicho país, reafirmando el efecto de las imperfecciones en el mercado de capitales sobre el consumo y su relación con el exceso de sensibilidad.

En un análisis que involucra varios países Carroll y Summers (1989) previamente comprobaron que en el Japón efectivamente se observaban incrementos considerables en el consumo ante crecimientos importantes en el ingreso, de forma alternativa en aquellos países donde el ingreso crecía poco, los incrementos del consumo eran menores. Este comportamiento según los autores podría estar vinculado a alguna causa común que afecta de forma paralela el crecimiento del ingreso y el consumo, o al hecho de que efectivamente los consumidores resultan más sensibles al ingreso corriente de lo que predeciría la HIPER

Algunas regularidades los llevan a inclinarse por esta última explicación. Por ejemplo, es de esperar que cuando se suaviza el consumo, reducciones en la tasa de crecimiento futura, lleven a que los países incrementen sus tasas de ahorro. Ello contrasta con has tasas de ahorro relativamente estables observadas en los diversos países.

Si bien en diferentes casos puede corroborarse cierto grado de sensibilidad del ingreso al consumo, ello no implica que los individuos actúen irracionalmente, o que omitan la intertemporalidad en la toma de sus decisiones. Las múltiples restricciones, los hábitos u otros aspectos, implican $\mathrm{b}$ coexistencia de individuos diversos, y algunas regularidades empíricas pueden ser explicadas cuando no se adopta una representación excluyente.

Muestra de ello es el trabajo de Campbell y Mankiw (1989) quienes al caracterizar simultáneamente dos tipos de consumidores, unos que actúan de acuerdo a la HIPER y otros que se guían por la evolución del ingreso corriente, logran retener ciertos hechos de la economía de los Estados Unidos.

\footnotetext{
${ }^{13}$ Wakabayashi y Horioka (2005), Borrowing Constraints and Consumption Behavior in Japan, NBER Working Paper No. 11560.

${ }^{14}$ Medida a través de diversos indicadores como acceso a tarjetas de crédito.
} 
Dentro de estos hechos, y en contraste con la versión simple de la hipótesis del ingreso permanente, comprueban que el pronóstico del consumo no se restringe a una caminata aleatoria puesto que estiman, a partir de su muestra ${ }^{15}$, que cuando se prevé un incremento del $1 \%$ en el ingreso se puede esperar un incremento del $0.5 \%$ en el consumo. Simultáneamente periodos en los cuales el consumo es relativamente alto en comparación con el ingreso, suelen ser seguidos por periodos donde el ingreso crece de manera importante, sugiriendo un comportamiento compatible con la formación de expectativas racionales.

\subsection{LA EVOLUCIÓN DEL PRONÓSTICO COMO APORTE A LA DISCUSIÓN TEÓRICA}

La concentración en la bondad del pronóstico y su evolución frente a cierto rango de choques $^{16}$ ha llevado a desestimar erróneamente algunas estimaciones. Como anota Eitrheim et al (2000) ${ }^{17}$, una estrategia que pone mucho énfasis en el comportamiento del pronóstico y sus fallas, sin una evaluación cuidadosa de las causas, corre el riesgo de desestimar modelos que pueden contener importantes elementos estructurales.

Los errores en el pronóstico son frecuentemente causados por cambios en los parámetros no estructurales. Por ejemplo Doornik y Hendry (1997) y Clements y Hendry (1999) muestran que la principal fuente de falla en el pronóstico son cambios determinísticos en la media de equilibrio (ejemplo, la tasa de ahorro de equilibrio) más no cambios en los coeficientes derivados (ejemplo, propensión a consumir) que finalmente son los de interés para el análisis de política.

Ahora, si bien lo hechos confirman que un episodio de falla en el pronóstico no invalida la teoría subyacente ni valida su rival, lo cierto es que a partir de la hipótesis del ingreso permanente con expectativas racionales (HIPER) se dedujo que el mejor predictor del

\footnotetext{
${ }^{15}$ Que comprende información trimestral desde 1953 hasta 1986.

${ }^{16}$ Impuestos y cambios en el presupuesto, cambios en la tasa de interés, tasas de desempleo etc.

${ }^{17}$ Eitrheim O. et al. (2000) "Progress from forecast failure-The Norwegian consumption function". Norges Bank.
} 
consumo era el propio consumo rezagado y que ninguna otra variable podría predecir mejor el consumo presente.

Esta fuerte afirmación esta sujeta a revisión de observarse un mayor ac ierto en el pronóstico originado a partir de una estimación que involucre variables asociadas a un enfoque teórico alternativo a la HIPER. Si la proyección que se deriva de este último supera el pronóstico de una caminata aleatoria, ello resulta suficiente para refutar la afirmación de que el consumo pasado es el mejor predictor de él mismo.

En este punto h bondad del pronóstico constituye un referente para discriminar la teoría subyacente. Obsérvese que si el mejor predictor del consumo no es el propio consumo rezagado se estaría inclinado por afirmar que a pesar de la HIPER, cuestiones como las restricciones crediticias, los hábitos etc., podrían estar condicionando la evolución del consumo y por tanto a partir de una caminata aleatoria no se obtendría necesariamente la mejor predicción.

Un error en el pronóstico no constituye una condición suficiente para desestimar una teoría, en tanto que un mayor ajuste en comparación a una caminata aleatoria ciertamente contradice que esta última sea la mejor opción para determinar la trayectoria futura del consumo.

\section{CORROBORACIÓN EMPÍRICA - EL CASO COLOMBIANO}

Con el objetivo de aproximarse a la definición de los determinantes macroeconómicos del consumo de los hogares para el caso colombiano es pertinente evaluar en primera instancia la relación más simple, sugerida por Keynes (1936), y dada por aquella entre el ingreso disponible y el consumo.

De acuerdo a las gráficas del anexo 3a, y como era de esperarse, se observa una relación positiva entre el crecimiento anual del consumo de los hogares y el crecimiento anual del 
ingreso, medido como el ingreso salarial o el ingreso disponible ${ }^{18}$. Resulta sin embargo menos dispersa la relación cuando se considera el ingreso bajo esta última definición.

Retomando el argumento de Campbell y Mankiw (1989) según el cual, bajo expectativas racionales, periodos en los cuales el consumo es relativamente alto son seguidos por periodos donde el ingreso crece de manera importante, resulta interesante comparar de forma preliminar dos tipos de relaciones. Primero aquella relación dada entre el crecimiento del consumo del año $t-1$ y el crecimiento del ingreso en el año $t$ y segundo la relación que surge entre el crecimiento del consumo en el año $t$ y el crecimiento del ingreso en el año anterior. Como se aprecia en las gráficas existe una relación más estrecha en el caso de la última relación. Este hecho, a primera vista, sopesaría más el efecto del ingreso sobre el consumo y menos el comportamiento esperado bajo expectativas racionales.

Dentro de las estimaciones adelantas para Colombia de la relación entre el consumo y el ingreso puede encontrarse la elaborada por Carrasquilla (1989) quien a través de una serie de regresiones concluye que la implicación estocástica de la no existencia de predictores significativos del consumo agregado, asociada a la HIPER, es violada en el caso colombiano en la medida que se atribuye cierta influencia del ingreso sobre el consumo. Se pregunta sin embargo si esto es suficiente para descartar la HIPER más aún cuando la significancia de los parámetros por él estimados puede ser espurea en la medida que los residuos asociados a su estimación no se juzgan como ruido blanco.

Al corroborar que el flujo de consumo exhibe una raíz unitaria y que el PIB también exhibe un comportamiento asimilable a un paseo aleatorio prueba que las tendencias aleatorias encontradas de manera individual son en realidad una única tendencia común, asociadas al hallazgo de un vector de cointegración. Finalmente encuentra que la HIPER es válida "tanto por el comportamiento del consumo, como por la existencia de cointegración entre C y $\mathrm{Y}^{, 19}$ y opera como una relación de largo plazo a la cual tienden a ajustarse los choques temporales que sufre la economía.

\footnotetext{
${ }^{18}$ Una descripción mas detallada de los datos y sus fuentes se observa en el anexo 2.

19 Carrasquilla, A (1989). "La Asignación Intertemporal del Consumo en Colombia: Un enfoque de Cointegración”, ESPE Banco de la República.
} 
Afirmar que la existencia de cointegración valida la HIPER no es suficiente. De hecho, en caso de hacerse para cierto periodo la respectiva evaluación del pronóstico y corroborar que la senda de consumo que se pronostica a partir de una estimación particular (que puede incluir además del ingreso y del término de corrección de error, variables plausibles como el acceso al crédito, la riqueza etc.) supera en bondad de ajuste al consumo rezagado (mejor predictor de una caminata aleatoria), la conclusión de la HIPER en relación al pronóstico no aplicaría para el periodo evaluado.

A pesar de ello, la técnica de estimación implementada por Carrasquilla (1989), quien considera un sistema de información que involucra el término de perturbación de largo plazo, va en la dirección correcta si se tiene en cuenta que el objetivo es contrastar las predicciones de largo plazo, y como anotan Carroll y Summers (1989) basta con adoptar una técnica que haga explicita esta relación de largo plazo. De no ser así se correría el riesgo de concentrarse en las fluctuaciones del consumo y el ingreso y su relación con en el problema de la substitución entre renta presente y futura, y se terminaría abordando la cuestión del consumo desde una perspectiva de corto plazo.

Por ende, la presente revisión de los determinantes macroeconómicos de largo plazo del consumo total de los hogares en Colombia, y su posible variación tras la evolución del ciclo económico en los últimos años, se respalda empíricamente en el análisis de estimaciones que incluyen de igual forma dicho componente de largo plazo ${ }^{20}$.

En contraste con los hallazgos de Carrasquilla (1989), si se plantea un modelo bivariado que involucra las variables sugeridas por la HIA, y en particular el consumo y el ingreso disponible $^{21}$ se encuentra que la relación de largo plazo no afecta la dinámica del consumo. Si se modifica el sistema bivariado manteniendo el consumo per. capita de los hogares pero involucrando el ingreso salarial no se encuentra siquiera una relación de largo plazo, lo que

\footnotetext{
${ }^{20}$ Para ahondar en la técnica de estimación revísese el anexo 4.

21 Para todas las estimaciones se tratan las variables en términos per. capita. Valga anotar que las mismas también se evaluaron en niveles agregados sin encontrarse ningún tipo de relación.
} 
lleva a afirmar que no es posible establecer una relación estructural entre el consumo y el ingreso bajo esta representación ${ }^{22}$.

Retomando el hecho verificado para otras estimaciones donde h inclusión de variables adicionales que pretenden cuantificar el bienestar de los hogares ha resultado determinante en la posibilidad de establecer una relación más precisa y estable, varios autores han coincidido en tomar como proxy de la riqueza de los hogares variables asociadas con uno de sus activos más importantes, la vivienda.

De acuerdo a la representación gráfica del anexo 3b, la volatilidad del crecimiento anual del consumo total de los hogares es mayor que la del stock de vivienda. Aún así obsérvese que periodos donde asciende o desciende el stock de vivienda coinciden con periodos de expansión o contracción del consumo total de los hogares sobre todo a partir de la década de los 70 .

Dentro de las estimaciones relacionadas, Duarte (2003) ${ }^{23}$ analiza la respuesta del consumo a cambios en la riqueza. En línea con la HIP, plantea un modelo donde el consumo resulta proporcional a la riqueza total, compuesta a su vez de la riqueza no humana y h riqueza humana, menos un término de perturbación asociado con la incertidumbre.

Duarte (2003) supone, para el periodo 1987-2000, que la riqueza humana es proporcional al PIB, toma como proxy de la riqueza no humana el precio real de la vivienda y como proxy de la incertidumbre de los activos la inflación del IPC. Una representación uniecuacional que incorpora el término de corrección de errores lo lleva a calcular que la elasticidad del consumo agregado respecto al precio de la vivienda es de $17 \%$. Encue ntra adicionalmente un efecto negativo de la tasa de interés sobre el consumo. Un punto porcentual de la tasa de interés reduce en 0.13 puntos porcentuales el consumo.

\footnotetext{
${ }^{22}$ Para una lectura más clara de los resultados en el anexo 5 se presenta el resumen de las distintas estimaciones.

${ }^{23}$ Duarte, L (2003). "Los determinantes del Consumo y la Propensión Marginal a la Riqueza en Colombia". Dirección General de Política Macroeconómica.
} 
Concluye que en Colombia se cumple parcialmente la HIPER, ya que el consumo planeado es función de la riqueza humana. Atribuye este hecho a la posible violación de supuestos del modelo como el horizonte de planeación finito, herencias o restricciones de liquidez. Reconoce además que errores de medición en variables como el precio de la vivienda pueden influir en la calidad de la estimación. La ausencia del análisis del pronóstico no le permite concluir sobre este frente y por ende dar respuestas más contundentes en relación a la pertinencia de uno u otro enfoque teórico.

Al revisar la relación e incorporar la historia reciente, considerando para ello un sistema trivariado que incluye junto con el consumo de los hogares y el ingreso disponible, el stock de vivienda como proxy de la riqueza de los hogares, se obtiene un modelo ${ }^{24}$ que cumple satisfactoriamente con todos los requerimientos del análisis de cointegración multivariado $^{25}$. Cabe anotar que cuando se toma la vivienda como variable proxy de la riqueza sería preferible considerar no solo las variaciones en cantidades sino también en precios relativos, sin embargo como reconoce Duarte (2003) las dificultades en la medición del precio de la vivienda afectan la calidad de las estimaciones.

En particular se observa que en el largo plazo el efecto sobre el consumo del crecimiento del stock de vivienda es claramente superior al efecto de las variaciones en el ingreso disponible. Para esta caracterización cuantitativa de las relaciones es preciso mencionar que no es prudente obtener las elasticidades de largo plazo a partir del vector de cointegración. Como señala Misas et al (2001), citando a Johansen, esta interpretación es discutible al prescindir del componente de corto plazo. Por lo tanto las elasticidades se obtienen partiendo de la función impulso respuesta del sistema o de su representación $M A^{26}$.

Siendo así y según se aprecia en el anexo 6, la variación en el consumo de los hogares ante incrementos en el stock de vivienda sería de 3.82, 3.487 o 4.26, según se lea este efecto de largo plazo a partir de la matriz $\mathrm{C}$ (que surge de la representación $M A$ ), del efecto

\footnotetext{
${ }^{24}$ Con tendencia en el vector de cointegración.

25 En este caso existe evidencia de exogeneidad débil del ingreso disponible lo que lleva a imponer la respectiva restricción.

${ }^{26}$ MA: Moving average. Al respecto revísese el anexo 4.
} 
acumulado de las primeras diferencias de la función impulso respuesta o de la reacción de largo plazo en la función de impulso respuesta. Entre tanto la elasticidad del ingreso sería de $0.106,0.39$ o 0.78 respectivamente ${ }^{27}$.

Para evaluar el efecto de la tasa de interés se procede a adicionar esta variable al anterior sistema de información ${ }^{28}$. El impacto de una variación en la riqueza disminuye, en tanto que el efecto del ingreso disponible sobre el consumo aumenta, aunque el primero sigue siendo superior comparado con el segundo independientemente de la forma como se obtengan las elasticidades. En el largo plazo la elasticidad del consumo per cápita a la tasa de interés sería $-0.11,-0.158$ o - 0.047 según la lectura que se haga de la misma ${ }^{29}$.

Incorporando variables asociadas a la estructura demográfica del país, compatibles con la $\mathrm{HCV}$, estudios anteriores han revelado su poca relevancia en la determinación de los patrones de consumo y ahorro para el caso colombiano, véase por ejemplo López et al $(1996)^{30}$. De hecho y según se observa en el anexo 3c, no es clara la relación del crecimiento anual del consumo total de los hogares y el crecimiento anual de la dependencia demográfica, medida como la proporción de la población en un rango de edades que se pudiera entender productivo en relación a la población total. El crecimiento del consumo total de los hogares es mucho más volátil que las variaciones en la estructura demográfica.

De hecho, según se observa en la tabla resumen de las estimaciones (anexo 5), considerando la variable de dependencia demográfica como la proporción de la población entre 20 y 64 años no se logra establecer una relación de largo plazo ${ }^{31}$. Aún si se modifica

\footnotetext{
27 También se contempló en el sistema trivariado el ingreso salarial envés del disponible, pero según se observa en el anexo 5 los resultados fueron menos satisfactorios. En particular la consistencia de los signos de la relación de largo plazo no es la esperada.

${ }^{28}$ Tras el análisis se mantiene el modelo tipo cidrift (2) y también se encuentra evidencia para imponer la restricción de exogeneidad débil sobre el ingreso disponible.

${ }^{29}$ Nuevamente se plantea una variante del sistema con el ingreso salarial. La exclusión de la riqueza y del consumo del vector de cointegración llevan a descartar esta especificación.

30 López, Misas y Oliveros (1996). "Understanding Consumption in Colombia" Banco de la República. Borradores de Economía No. 58.

31 Esta variable se contempla dentro de diversas variantes trivariadas, tetravariadas y pentavariadas, que adicionalmente consideran el ingreso salarial o el ingreso disponible.
} 
la tasa de dependencia y se tiene en cuenta la proporción de la población entre 30 y 64 años o 40 y 64 años no se logra establecer dicha relación.

El trabajo de López et al. (1996) además de la demografía evalúa diferentes sistemas con información comprendida entre 1953 y 1993. También bajo la técnica de cointegración, en su estimación del equilibrio de largo plazo incorporan junto con el ingreso laboral, la riqueza y la tasa de interés, algunas variables relacionadas con la crítica a la HIPER como son las restricciones crediticias y la volatilidad del ingreso.

Conciben en consecuencia cinco sistemas que adicionan a las variables anteriores (riqueza, tasa de interés e ingreso laboral), proxies de restricciones crediticias, incertidumbre en el ingreso, utilidad derivada del consumo público y privado y estructura demográfica ${ }^{32}$. Encuentran que los sistemas que adicionan el consumo público y la volatilidad del ingreso son los que mejor satisfacen los criterios del análisis de cointegración.

Concluyen que el ingreso laboral es el mayor determinante del consumo total de bs hogares en el corto plazo. Según el mecanismo de corrección de error el consumo crecería entre $0.66 \%$ y $0.7 \%$ ante un aumento de $1 \%$ en el ingreso. Además existe un efecto negativo de la tasa de interés sobre el consumo. En el largo plazo el consumo per. cápita se incrementa entre $0.6 \%$ y $1 \%$ si la tasa de interés decrece en un punto. El trabajo encuentra adicionalmente escasa evidencia que sugiriera que las restricciones crediticias hicieran parte de alguna relación de largo plazo.

Como se anotó anteriorme nte la interpretación de las elasticidades de largo plazo basándose en los coeficientes estimados para el vector de cointegración no resulta del todo precisa. Adicionalmente, y de forma similar a Carrasquilla (1989) y Duarte (2003), la ausencia de la evaluación de la bondad de pronóstico de los sistemas estimados no permite decir mucho acerca de la pertinencia de la HIPER.

\footnotetext{
32 En su orden las proxies correspondientes son: crédito al sector privado como proporción del PIB, desviación estándar del ingreso laboral tres años atrás, consumo público real per. capita, consumo privado real per. capita y porcentaje de la población entre los 18 y los 64 años de edad.
} 
Un punto que merece especial atención es la irrelevancia, que para el periodo de estudio de López et al (1996) tienen las restricciones crediticias. Esta situación puede diferir de estimaciones más recientes de acuerdo al importante cambio que se observa en algunas de las variables que sirven como proxies de acceso al crédito. Según se aprecia en el anexo 3d la relación Crédito/PIB después de 1997 desciende inclusive a pesar de la disminución en el denominador, que cae por efecto del retroceso en el crecimiento del producto tras la recesión de 1998.

Concentrándose en la última década y como lo revela la gráfica del anexo $3 \mathrm{~d}$, la intermediación financiera en Colombia se estancó tras la crisis de 1998 alrededor de una razón crédito PIB del $30 \%$ en promedio ${ }^{33}$. Según un informe del Banco Mundial ${ }^{34}$ este nivel está significativamente por debajo de las razones observadas para Chile, a la par con los de México y por encima de las relaciones calculadas para el Perú. El descenso en la intermediación por parte de la banca colombiana puede atribuirse a una creciente aversión al riesgo tras la crisis bancaria de 1998 .

El deterioro en la intermediación se asocia también a una mayor exposición de los bancos a los bonos y prestamos hacia sector público que termina desplazando el crédito destinado al sector privado (anexo 3d). La profundización financiera relativamente baja comparada con otros países de la región, y que se mantiene hasta 2002 en niveles inferiores al periodo que antecede la crisis, repercute en el acceso al crédito. Descensos en el número de cuentas corrientes y de ahorros tras la crisis así lo indican(anexo 3d).

Respaldando la importancia del acceso al crédito resulta interesante observar (también en el anexo 3d) la relación que guarda el consumo total y de bienes durables por parte de los hogares con las ventas y avances con tarjeta de crédito ${ }^{35}$. En comparación con el consumo total se percibe una relación más evidente entre el consumo de bienes durables por parte de los hogares y está medida aproximada de acceso al crédito.

\footnotetext{
${ }^{33}$ Hasta el año 2002.

${ }^{34}$ Solo y Manroth (2006) “Access to Financial Services in Colombia” World Bank Policy Research Working Paper 3834.

${ }^{35}$ Información con periodicidad trimestral.
} 
Incorporando la historia reciente y considerando junto con el consumo de los hogares y el ingreso disponible, la cartera total del sistema financiero como proporción del $\mathrm{PIB}^{36}$, es posible establecer de hecho una relación de largo plazo. Comparando las elasticidades con las obtenidas en las actualizaciones presentadas previamente se observa un mayor efecto del ingreso sobre el consumo. De hecho cuando la variable proxy de profundización financiera hace parte de la especificación, la elasticidad ingreso aumenta independientemente de la lectura que se haga de la misma (1.12, 0.75 o 1.08, anexo 6).

Un mayor acceso al crédito implica la posibilidad de suavizar el consumo. Es decir si se sabe que el ingreso corriente es inferior al ingreso permanente, eventualmente el consumo corriente puede estar por encima del ingreso de ese periodo y viceversa. Por tal motivo, el sentido del efecto sobre el nivel del consumo no es unidireccional, en tanto que si es claro que un mayor acceso al crédito representa una menor volatilidad del consumo. El punto al contemplar la proxy de acceso al crédito esta en la relevancia que cobra el ingreso corriente como determinante del consumo ${ }^{37}$ y que se ratifica empíricamente para el caso colombiano.

De acuerdo a las elasticidades halladas para Colombia el efecto de largo plazo de la profundización financiera sobre el consumo total de los hogares es negativo (-0.16, - 0.19 o -0.15). Por tanto, a un horizonte prolongado y dado un nivel de ingreso, los hogares ahorran más y consumen menos entre mayor acceso al crédito tengan.

\subsection{EVALUACIÓN DEL PRONÓSTICO PARA LOS SISTEMAS ENCONTRADOS}

Un punto relevante y no evaluado en los trabajos anteriores es el juzgamiento de la bondad del pronóstico. Se realiza en consecuencia una evaluación sobre la calidad del mismo basado en aquellas estimaciones que cumplieron satisfactoriamente con los criterios del análisis de cointegración.

\footnotetext{
${ }^{36}$ Obsérvese que se trata de una proxy más general a la utilizada por López et al. (1996).

37 Al considerar conjuntamente las variables que resultaron relevantes: consumo de los hogares, ingreso disponible, riqueza, tasa de interés y restricciones crediticias ciertos criterios del análisis de cointegración son insuficientes. Véase anexo 5.
} 
La evaluación de pronóstico que se sigue es de tipo rolling. Esta consiste en realizar el pronóstico manteniendo el tipo de modelo (drift o cidrift) y la estructura de rezagos hallada inicialmente con información a 2002, pero actualizando la estimación de los parámetros de corto y largo plazo al incluir progresivamente información hasta los años 1997, 1998, 1999, 2000 y 2001. De observarse que ha dichos periodos el tipo de modelo y la estructura de rezagos varía, se toma la mejor especificación a cada uno de los respectivos años. Posteriormente se calculan y promedian algunas de las medidas estándar utilizadas para la evaluación de pronóstico ${ }^{38}$ a un horizonte de tiempo similar ${ }^{39}$.

Una medida de bondad del pronóstico que cobra especial relevancia por su aporte en la discusión teórica es la $\mathrm{U}$ de Theil. De acuerdo a esta sencilla razón entre el error de pronóstico de un modelo en particular y el error de pronóstico de una caminata aleatoria, de observarse una relación inferior a uno es posible concluir que el pronóstico del modelo alternativo supera el de una caminata aleatoria.

Bajo la consideración que la falla en el mismo no necesariamente implica que los coeficientes de largo plazo sean incorrectos, cuando se observan los distintos criterios de pronóstico en los sistemas de información hasta ahora estimados sobresale que la $\mathrm{U}$ de Theil es superior a uno (véase anexo 8).

Ello sugiere que a los distintos horizontes evaluados y frente a los pronósticos de las especificaciones contempladas, el mejor predictor del consumo total per. cápita de los hogares es el propio consumo en el presente. Las conclusiones de la HIPER corresponderían con la evaluación del pronóstico para estos casos y por tanto, aunque existe evidencia de la relación de largo plazo, los modelos considerados no son suficientes para poder determinar la evolución del consumo de los hogares.

\footnotetext{
${ }^{38}$ La forma en que se construyen las diferentes medidas de evaluación se resume en el anexo 7.

${ }^{39}$ Siendo las medidas a un horizonte de cinco años aquellas que se derivan del pronóstico hasta 2002 con información a 1997.
} 
No obstante, si bien a primera vista la bondad del pronóstico para el sistema que involucra la variable de profundización financiera revela que el mejor predictor del consumo es el propio consumo rezagado, se aprecia una mejora importante en la $\mathrm{U}$ de Theil en relación a las otras estimaciones. Inclusive a un horizonte de cuatro años el pronóstico a partir del VEC estimado para este sistema de información supera el de una camita aleatoria.

Realizando una observación más detallada y por años del ejercicio de evaluación fuera de muestra (anexo 8) para este sistema de información, se aprecia que sobre la U de Theil promedio inciden particularmente los errores en el pronóstico de los modelos estimados con información hasta 1999 y 2000; años ciertamente atípicos dado que en 1999 el crecimiento del producto estuvo alrededor del $-4 \%$.

En contraste, si se hubiese hecho el pronóstico con información hasta 1997, 1998 o 2001, una caminata aleatoria no hubiera superado al pronóstico que se estima a partir del modelo de corrección de errores. Según se anotó en el apartado 3.1, un episodio atípico como el observado en 1999 puede implicar un cambio determinístico importante en la media de equilibrio, y en consecuencia producir errores de pronóstico, pero no necesariamente cambios en los coeficientes derivados para el largo plazo.

Adicionalmente, no es suficiente que en ciertos periodos la $U$ de Theil revele que la caminata aleatoria es la mejor alternativa de pronóstico para afirmar que las conclusiones de Hall en lo referente al mejor predictor del consumo sean ciertas. La posibilidad de que el desacierto en el pronóstico se deba entre otras a la omisión de alguna o algunas variables persiste. El punto es que para aquellos periodos donde la especificación sugerida supera la caminata aleatoria, no hay duda de que esta última no es la mejor opción de predicción. La bondad de ajuste del pronóstico puede ser susceptible de mejorar.

Valga precisar que se probó que el tipo de especificación inicialmente hallada con información hasta 2002 (que involucra una tendencia en el vector de cointegración y requiere de dos rezagos) se mantiene en cada uno de los periodos analizados. La estabilidad en el vector de cointegración se muestra en el anexo 9. 


\subsection{CONTEXTUALIZACIÓN DE ALGUNOS HALLAZGOS EMPÍRICOS}

El efecto negativo de la profundización financiera sobre el consumo total se atribuye a que en el largo plazo dado un mayor acceso al sistema financiero los hogares ahorran más y consumen menos. Por ejemplo es muy factible que un hogar colombiano, al cual se le abre el acceso a la financiación destine buena parte de su ingreso al pago de una cuota hipotecaria. Cabe anotar sin embargo, que en el corto plazo, algunos autores encuentran un efecto positivo de la profundización financiera sobre el consumo. Al respecto puede revisarse la estimación hecha por Bennet, Loayza y Hebbel (2001) para el caso chileno.

Como indican Loayza, Hebbel y Servén (2001) la liberalización financiera abarca un conjunto de medidas como la eliminación de los límites al crédito, relajamiento de los requisitos a la entrada a instituciones financieras extranjeras, liberalización de tasas de interés, desarrollo de mercados de capital y el fortalecimiento de la regulación prudencial y de la supervisión. Analíticamente el efecto de la liberalización se puede dividir en un efecto directo, con un impacto positivo sobre el consumo en el corto plazo, y otro indirecto con efectos positivos sobre el ahorro hacia el largo plazo.

El impacto directo de corto plazo está además constituido por canales de precio y cantidad. El primero opera vía tasas de interés. Tras la disminución en la tasa de interés (una vez se amplia la oferta de crédito) y dado que, como se probó en Colombia, prima el efecto sustitución sobre el ingreso, es de esperar que aumente el consumo y disminuya el ahorro. Adicionalmente el hecho de que se amplíe el acceso de los hogares al crédito hace que sean capaces de financiar mayores niveles de consumo dados sus niveles de ingreso ${ }^{40}$.

Hacia el largo plazo Loayza y Shankar (2000) sugieren que, en países como la India, la liberalización de los mercados financieros domésticos y el fortalecimiento del sector

\footnotetext{
${ }^{40}$ El componente de corto plazo de la estimación actualizada para Colombia respalda el impacto positivo que tiene inicialmente el mayor acceso al crédito sobre el consumo. Según se observa en la ecuación de la primera diferencia del logaritmo natural del consumo total de los hogares, el coeficiente estimado del rezago anual de la primera diferencia del logaritmo natural del crédito en proporción al PIB es positivo, significativo e igual a 0.1265 .
} 
bancario han inducido a que los agentes privados cambien la composición de sus activos en favor de bienes durables. Los autores van más allá y proponen, para el caso de la India, incluir en la contabilidad del ahorro el gasto en bienes durables.

El anterior patrón de gasto dado un mayor acceso al crédito se puede extender para el caso colombiano. Recopilando la regularidad observada en el anexo $3 \mathrm{~d}$, puede verse como la dinámica en las ventas y avances con tarjeta de crédito está mucho más relacionada con la evolución en el consumo de durables que con la tendencia en el consumo de bienes totales por parte de los hogares. De hecho siguiendo esta preferencia por durables una vez se cuenta con el suficiente acceso al crédito puede que los hogares destinen parte de su ingreso al ahorro con el fin de invertir posteriormente en activos como la vivienda. Resulta coherente pensar que en el largo plazo entre mayores sean las restricciones y por tanto menor la participación del crédito, la porción del ingreso destinado a ahorrar sea menor.

Ante el particular comportamiento del gasto en bienes durables se abordó el problema por tipo de bienes. Para ello se analizaron dos conjuntos de datos. El primero con periodicidad anual comprendido entre 1965 y 2002, el segundo con información trimestral desde 1996:I hasta 2005:IV. Bajo este última frecuencia el periodo considerado es relativamente corto dada la disponibilidad de información, sin embargo se cuenta con medidas más especificas de restricciones crediticias, tal es el caso de las ventas y avances con tarjeta de crédito ${ }^{41}$.

Con el conjunto de información anual no se puede establecer una relación de largo plazo entre el gasto en bienes durables, las diversas medidas de ingreso y una proxy tan amplia de restricciones crediticias. La exclusión del ingreso de la relación de largo plazo es una constante en los diversos sistemas analizados.

Para el caso trimestral persiste la evidencia de exclusión del ingreso disponible de la relación de largo plazo por lo que se procede a estimar preliminarmente un sistema multivariado sin dicha relación. La estimación VAR, así como las representaciones uniecuacionales en aquellos casos donde no se puede rechazar la hipótesis de que la

${ }^{41}$ La descripción de los datos trimestrales se encuentra en el anexo 2. 
primera diferencia del logaritmo del consumo de durables no causa Granger la primera diferencia del logaritmo del ingreso disponible, no arroja estimaciones satisfactorias en términos de significancia y comportamiento de los residuales. La brevedad de la información puede estar incidiendo.

\section{CONCLUSIONES}

La cuestión del consumo y sus determinantes en Colombia no es para nada un tema nuevo. De hecho, el caso colombiano coincide con la multiplicidad de estimaciones observadas en la literatura general

El problema es un asunto vigente pues queda claro, tras la presente revisión, que al pretender caracterizar la evolución del consumo es necesario investigar en determinados periodos las relaciones que avalan su dinámica. Por ejemplo, al incorporar la historia reciente de la economía colombiana, las restricciones crediticias terminan jugando un papel importante en la explicación de su comportamiento, lo cual contrasta con estudios precedentes.

Las estimaciones corroboran adicionalmente para el caso colombiano la existencia de una relación de largo plazo entre el consumo de los hogares, la proxy de riqueza y la tasa de interés. La dependencia demográfica no es una variable que afecte los patrones de ahorro y consumo para Colombia lo que concuerda con hallazgos previos.

El efecto del stock de vivienda, como proxy de la riqueza, supera sustancialmente el efecto del ingreso disponible sobre el consumo de los hogares. En relación a la tasa de interés las elasticidades estimadas avalan para el caso colombiano un efecto negativo sobre el consumo y en términos cuantitativos corresponde con algunas estimaciones anteriores (en promedio -0.105).

Pese a la existencia de la relación de largo plazo en diversos sistemas de información, el ingreso disponible podría ser una variable útil en la predicción del consumo total de los 
hogares solo cuando se consideran paralelamente las restricciones crediticias (salvo aquellos periodos atípicos donde el ingreso experimenta serias fluctuaciones). La bondad de ajuste deficiente en sistemas que cumplen con los criterios del análisis de cointegración no constituye una razón suficiente para desestimar las elasticidades de largo plazo. Como se menciono, una estrategia que pone mucho énfasis en el comportamiento del pronóstico y sus fallas, sin una evaluación cuidadosa de las causas, corre el riesgo de descartar modelos que pueden contener importantes elementos estructurales.

La relevancia que toma el ingreso al considerar simultáneamente el acceso al crédito sugiere que no se da una completa suavización de la trayectoria de consumo al verse limitada esta posibilidad La mayor elasticidad del consumo frente al ingreso, cuando se controla por la presencia de restricciones al crédito, respalda este aumento en la sensibilidad del consumo.

El hecho de que el ingreso corriente de los hogares afecte la evolución del consumo no implica que estos actúen de manera irracional. Las restricciones al crédito, acentuadas tras la crisis de 1998, limitan la capacidad de flexibilizar el gasto. Hacia el largo plazo cuando los hogares gozan de mayor acceso al sistema financiero, terminan destinando una creciente proporción de su ingreso a ahorrar. Casos externos sugieren que dado este mayor acceso, los hogares tienden a acumular bienes durables y activos como la vivienda. Es por tanto recomendable ahondar en la identificación de las restricciones que imperan en Colombia.

Al tratar de ser más específicos, y considerando la amplitud de una variable como el consumo, se desarrollaron diversas estimaciones para el caso del gasto en bienes durabes. Con periodicidad trimestral el periodo considerado es rela tivamente corto dada la disponibilidad de información, sin embargo se cuenta con medidas más especificas de restricciones crediticias, tal es el caso de las ventas y avances con tarjeta de crédito. Entre tanto con el conjunto de información anual no se puede establecer una relación de largo plazo entre el gasto en bienes durables, las diversas medidas de ingreso y una proxy tan amplia de restricciones crediticias. Cabría con el tiempo solucionar los problemas asociados a la brevedad y medición de la información. 
La tendencia por destinar mayor proporción del ingreso a la compra de durables (automóviles por ejemplo) y eventualmente al ahor ro para la adquisición de activos como la vivienda dado un mayor acceso al crédito, es una cuestión abierta. La transición del efecto positivo de corto plazo del acceso al crédito sobre el consumo total vs. el efecto positivo sobre el ahorro en el largo plazo puede ser eventualmente estudiado a través de modelos de umbral que determinen el momento y las variables cruciales en la transición. 


\section{BIBLIOGRAFÍA}

Bennett, H. et al. (2001) "Un estudio del Ahorro Agregado por Agentes Económicos en Chile" Análisis empírico del ahorro en Chile, editado por Morandé y Vergara. Banco Central de Chile.

Bernheim,B. et al. (1985) "The Strategic Bequest Motive", Journal of Political Economy, 93 (6).

Berg, L. (1995) "Housing and Financial Wealth; Financial Deregulation and Consumption - The Swedish Case: The experience of the Nordic countries. Scandinavian", Journal of Economics 97 (3).

Brodin, P. y R. Nymoen (1989) "The Consumption Function in Norway. Breakdown and Reconstruction” Arbeidsnotat 1989/7, Norges Bank.

Brodin, P. y R. Nymoen (1992) "Wealth Effects and Exogenity: The Norwegian Consumption Function 1966.1-1989.4", Oxford Bulletin of Economics and Statistics, 54. A

Camerer, C. y Chua, Z (2006) "Experiments on Intertemporal Consumption with Habit Formation and Social Learning", unpublished research paper.

Carrasquilla, A (1989). "La Asignación Intertemporal del Consumo en Colombia: Un enfoque de Cointegración”, ESPE Banco de la República.

Carrizosa (1985) "Las tasas de interés y el ahorro financiero en Colombia”, Rev. Banca y Finanzas, 189.

Carroll y Summers (1989) "Consumption Growth Parallels Income Growth: Some New Evidence”, NBER, WP No. 3090

Carroll, Christopher D. (1997) 'Buffer-Stock Saving and the Life Cycle/Permanent Income Hypothesis,' Quarterly Journal of Economics, 112(1), 1-56

Carroll, Christopher D., Jody Overland, and David N. Weil (2000). "Saving and Growth with Habit Formation" American Economic Review., 90 (3), 341-355.

Clements y Hendry (1999) "Forecasting Non-stationary Economic Time Series", The MIT Press, Cambridge.

Davidson, J. E, Hendry, D, Srba, F y Yeo, S (1978) "Econometric Modelling of The Aggregate Time-Series Relationship between Consumers" Economic Journal, 88

Deaton, A (1992) “Understanding Consumption”, Oxford University Press

Duarte, L. (2003) "Los determinantes del Consumo y la Propensión Marginal a la Riqueza en Colombia" Versión interna de la Dirección General de Política Económica del Ministerio de Hacienda. 
Doornik y Hendry (1997) "The Implications for Econometric Modelling of Forecast Failure" Scottish Journal of Political Economy.

Dusenberry, James S. (1949) "Income, Savings, and the Theory of Consumer Behavior" Cambridge MA: Harvard University Press.

Eitrheim O. et al. (2000) "Progress from forecast failure-The Norwegian consumption function”. Norges Bank.

Fernández - Corugedo E. (2004) "Consumption Theory". Handbooks in Central Banking. Bank of England.

Flavin, M. (1981) “The Adjustment of Consumption to Changing Expectations About Future Income”, Journal of Political Economy, 89.

Friedman, M (1957) "A Theory of the Consumption Function". Princeton University Press.

Gallego, Fet al (2001) "El Ahorro y el Consumo de Bienes Durables frente al ciclo económico ¿Consumismo, Frugalidad, Racionalidad?" Análisis empírico del ahorro en Chile, editado por Morandé y Vergara. Banco Central de Chile.

Gallego, F y Soto, R. (2000) "Evolución del Consumo y Compras de Bienes Durables en Chile, 1981-1999", Banco Central de Chile, Documentos de Trabajo, No. 79.

Hall, R. E. (1978), "Stochastic Implications of the Life Cycle-Permanent Income Hypothesis: Theory and Evidence" Journal of Political Economy, 86.

Harberger, Arnold (1969) "La tasa de Rendimiento de Capital en Colombia". Revista de Planeación y Desarrollo. Departamento Nacional de Planeación.

Hernández, JN (2005) 'Demanda de Importaciones para el Caso Colombiano: 19802004”. Borradores de Economía. Banco de la República.

Johansen, S. (1988), "Statistical analysis of cointegration vectors", Journal of Economic Dynamics and Control, No. 12, pages 231-54.

(1994), "The role of the constant and linear terms in cointegration analysis of non-stationary variables", Econometric Reviews, No. 13, pages 205-29.

Keynes, J. M- (1936), The General Theory of Employment, Interest and Money.

Kimball, M. S. (1990) "Precautionary saving in the small and in the large", Econometrica, 58

Loayza, N. et al (2001) "Una Revisión del Comportamiento y de los Determinantes del Ahorro en el Mundo" Análisis empírico del ahorro en Chile, editado por Morandé y Vergara. Banco Central de Chile. 
Loayza, N. y Shankar, R. (2000) "Private Saving in India", World Bank Economic Review, Vol 14 No. 3, pages 571-594.

López A, Gómez C y Rodríguez N. (1996) "La caída de la Tasa de Ahorro en Colombia durante los años noventa: Evidencia a partir de una Base de Datos para el periodo 1950 - 1993”. Borradores de Economía No. 57. Banco de la República.

Lucas, R. E. (1976) "Econometric Policy Evaluation: a Critique". The Phillips Curve and Labour Markets North Holland.

Lütkepohl, H. (1993), Introduction to Multiple Time Series Analysis, SprigerVerlag, Second Edition.

Misas et al (2001). "Exportaciones no tradicionales en Colombia y sus determinantes", Banco de la República, Borradores de Economía, No. 178.

Misas et al (1996) "Understanding Consumption in Colombia". Borradores de Economía No. 58.

Modigliani, F. (1949)"Fluctuations in the Saving-Income Ratio: A Problem in Economic Forecasting", Studies in Income and Wealth, 11, NBER.

Palumbo et al. (2002) 'On the Relationships between Real Consumption, Income, and Wealth"

Solo, M y Manroth, A. (2006) "Access to Financial Services in Colombia" World Bank Policy Research Working Paper 3834.

Wakabayasshi M y Yuji C. O. (2005) "Borrowing Constraints and Consumption Behavior in Japan”. NBER, WP No. 11560. 


\section{Anexo 1 \\ Implicaciones de las restricciones crediticias - Representación}

Suponga que el individuo enfrenta el problema de asignación del consumo para dos periodos y se encuentra en un mercado de capitales sin imperfecciones (caracterizado por aspectos como libre acceso al crédito y tasa de préstamo igual a tasa de depósito). En ese contexto la restricción que enfrenta el individuo a un horizonte de dos periodos vendría dada por la línea recta $A B$, donde se mantiene la relación $\left(c_{1}+\frac{c_{2}}{1+r}=y_{1}+\frac{y_{2}}{1+r}\right)$.

Gráfica A1

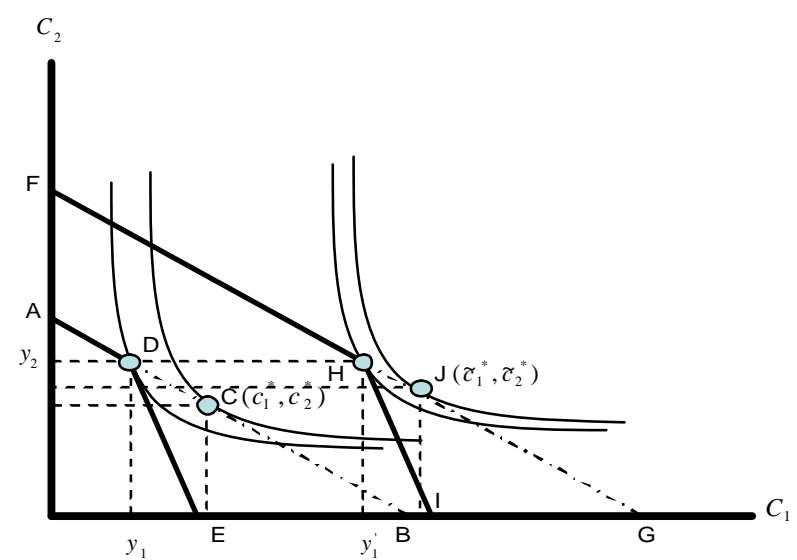

De acuerdo a su curva de indiferencia el individuo se ubicaría en el punto $C$ donde pide prestada una cantidad equivalente a $\left(c_{1}^{*}-y_{1}\right)$. Su consumo en el periodo 1 no se restringe por su ingreso corriente.

Considerando el caso en el cual la tasa de préstamo es superior a la de depósito (es decir, un tipo de imperfección en el mercado de capitales) la restricción del individuo vendría dada ahora por el segmento $A D E$. A la izquierda del punto $C_{1}=y_{1}$ la restricción vigente sería el 
segmento $A D\left(c_{1}+\frac{c_{2}}{1+r}=y_{1}+\frac{y_{2}}{1+r}\right)$ en tanto que a la derecha del punto $C_{1}=y_{1}$ la restricción sería aquella asociada al segmento $D E\left(c_{1}+\frac{c_{2}}{1+r^{\prime}}=y_{1}+\frac{y_{2}}{1+r^{\prime}}\right)$. El individuo maximiza su utilidad en el punto $D$, consumiendo todo su ingreso en cada periodo. La presencia de la imperfección hace que el individuo se ubique en un nivel de utilidad inferior a aquel que obtiene en el punto $C$.

Si el ingreso en el periodo 1 aumenta de $y_{1}$ a $y_{1}^{\prime}$ la restricción del individuo que no enfrenta restricciones crediticias pasa de la línea $A B$ a la línea $F G$, mientras que la restricción del individuo que enfrenta restricciones crediticias pasa de la línea $A D E$ a la línea $F H I$. El primero maximiza su utilidad en el punto $J\left(\widetilde{c}_{1}^{*}, \widetilde{c}_{2}^{*}\right)$ mientras que el último maximiza a un menor nivel de utilidad en el punto $H$.

Del ejercicio anterior queda claro que cambios en el consumo no corresponden estrictamente con cambios en el ingreso siempre que el individuo no enfrente restricciones crediticias (punto J). En el contexto de un mercado de capitales imperfecto, los cambios en el consumo coinciden con cambios en el ingreso corriente (punto $H$ ).

Este mismo ejemplo gráfico puede ser usado para analizar el caso en el cual el individuo no puede pedir prestado. En dicho caso la restricción incluiría al segmento AD pero el segmento DE sería vertical. 


\section{Anexo 2}

Datos

\section{Datos anuales}

El sistema de información anual que se analiza abarca observaciones per. capita comprendidas entre 1954 y el año 2002 y se construye a partir de las siguientes variables:

\section{Consumo}

Consumo de los Hogares (incluye ISFLSH ${ }^{42}$ ):

Para la construcción de la serie de consumo de los hogares de 1950 a 2004 en términos reales se toma en primera instancia la serie calculada por López et $\mathrm{al}^{43}$ (1996) del consumo nacional privado (de 1950 a 1990). La ventaja de esta serie es que utilizó un procedimiento que permitió tratar de forma conjunta las cuentas nacionales producidas por el DANE con aquellas series inicialmente calculadas por el Banco de la República para el periodo 1950 1980. De 1990 a 2004 la serie se actualiza con información del DANE.

\section{Consumo Final de Bienes Durables por parte de los Hogares:}

La serie de consumo de bienes durables para el periodo 1965 a 2002 en millones de pesos se construye en primera instancia para el periodo 1965 a 1995, según lo permite la discriminación disponible en la metodología base 75 del DANE, a partir de los crecimientos anuales en el consumo de muebles, accesorios fijos, alfombras y otras cubiertas para piso, aparatos de cocinar y calentar, refrigeradores, lavadoras y otros aparatos domésticos, aparatos y equipos terapéuticos, equipo de transporte personal y equipo y accesorios. Para el periodo 1992 a 2002 y con el cambio de metodología (base 94) ${ }^{44}$ se considera el consumo en muebles, accesorios, aparatos de cocinar y calentar, herramientas para la casa y el jardín, aparatos y equipos terapéuticos, compra de vehículos, equipos y accesorios, equipo para grabación de imagen y sonido, joyas y relojes (descontando las respectivas reparaciones). Para obtener la serie en términos reales el deflactor utilizado es el IPC fin de, base 1994.

\section{Ingreso}

Ingreso Disponible de los Hogares:

Para la serie del ingreso disponible en términos corrientes de 1950 a 2002 se toma de 1950 a 1987 el crecimiento anual del Ingreso Disponible Total de los Hogares en millones

\footnotetext{
${ }^{42}$ Instituciones sin fines de lucro que sirven a los hogares

${ }^{43}$ López A, Gómz C y Rodríguez N. La caída de la Tasa de Ahorro en Colombia durante los años noventa: Evidencia a partir de una Base de Datos para el periodo 1950 - 1993. Borradores de Economía No. 57 ${ }^{44}$ Estos cambios en metodología incluye aspectos como cambios en la mu estra y la redefinición de algunos ítems.
} 
de pesos calculado por López (1996). De 1988 a 2002 los crecimientos anuales del ingreso disponible de los hogares e instituciones privadas sin fines de lucro que sirven a los hogares según información del DANE.

\section{Ingresos Salariales:}

Para la construcción de la serie de ingresos salariales corrientes de 1950 a 2002 se considera de 1950 a 1987 el crecimiento anual de los ingresos salariales corrientes calculados por López (1996). De 1988 a 2002 se toma el crecimiento anual de la remuneración de los asalariados según un cuadro resumen de los agregados macroeconómicos del DANE.

\section{Población}

La proyección de la población colombiana parte de las observaciones puntuales de los censos de 1964 y 1973 y del dato de población de 1950 suministrado por el DNP. Desde 1985 se sigue la proyección del DANE. La dependencia demográfica se mide como la razón de la población entre $x$ y y años y la población total.

\section{$\underline{\text { Riqueza }}$}

\section{Stock de Vivienda:}

La serie del stock de vivienda se calcula partiendo de la formación bruta de capital físico en edificaciones residenciales y según la metodología de Harberger (1969) ${ }^{45}$ De 1950 a 1993 la serie de inversión en vivienda proviene de los cálculos de López et al (1996) y de 1994 en delante de información suministrada por la sección de Estadística del Banco de la República

\section{Interés}

De 1950 a1979 se toma la tasa de interés calculadas por Carrizosa (1985). Para el periodo 1980 a 1993 la información proviene de la tasa de los CDT'S 90 días en términos nominales según López et al (1996) y de 1994 en adelante la serie suministrada por el Departamento de Estadística del Banco de la República. El dato real se construye considerando la respectiva inflación anual.

\footnotetext{
${ }^{45}$ El método de Harberger acumula la inversión y descuenta la depreciación a un stock inicial de capital. El stock inicial siguiendo esta metodología se calculó como la razón entre el promedio de la formación bruta de capital entre 1950 a 1952 y la suma de un crecimiento promedio de la inversión en edificaciones para dicho periodo de 0.05 y una depreciación de la vivienda de 0.025 .
} 


\section{$\underline{\text { Restricciones Crediticias }}$}

Cartera total del Sistema Financiero como proporción del PIB:

La series de cartera total del sistema financiero y del PIB se extraen de la información contenida en la publicación de los Principales Indicadores Económicos 1923-1997 del Banco de la República De 1998 en adelante se utiliza la información de cartera neta suministrada por el Departamento Técnico y de Información Económica del Banco de la República. La serie del PIB para el periodo 1998 a 2002 proviene del DANE.

\section{Datos trimestrales}

\section{Consumo}

Consumo de Bienes Durables por parte de los Hogares:

Para la construcción de la serie trimestral de consumo de bienes durables en términos reales se toma la información estadística proveniente del DANE que desagrega el consumo final desestacionalizado de los hogares residentes efectuado dentro del territorio económico según tipo de bien para el periodo 1994:I a 2005:IV. De acuerdo a la clasificación del consumo según durabilidad, la metodología del DANE incluye dentro del grupo de durables los muebles, los aparatos de cocinar, las herramientas, quipos y aparatos terapéuticos, automóviles, relojes y joyas y equipo fotográfico.

\section{$\underline{\text { Ingreso }}$}

Ingreso Disponible con información de Balanza Pagos:

Dada la trimestralización de la cuenta corriente de 1996:I a 2005:IV la serie de ingreso disponible se construye para este mismo periodo agregando al producto interno bruto, la renta neta de los factores externos ${ }^{46}$ y las transferencias corrientes, estas últimas en una importante proporción constituidas por las remesas. Una variante de este cálculo del ingreso disponible se efectúa cuando al agregar al PIB la renta neta de los factores externos no se contemplan los ingresos y egresos que por intereses tiene el sector público, al tiempo que se descuenta la remisión neta de utilidades y dividendos y los intereses netos de la demanda externa privada. Los ingresos tributarios del gobierno nacional central ${ }^{47}$ se descuentan y se incluyen las transferencias corrientes.

\footnotetext{
${ }^{46}$ Ingresos menos egresos.

${ }^{47}$ Información proveniente de la sección de Sector Público del Banco de la República.
} 


\section{$\underline{\text { Restricciones Crediticias }}$}

Ventas y Avances con tarjetas de crédito :

La información de ventas y avances con tarjetas de crédito para el periodo 1996:I a 2005:IV proviene de la información mensual que reporta Redeban Multicolor, una compañía dedicada a la transferencia electrónica de fondos entre entidades financieras y establecimientos comerciales. 
Anexo 3a

Crecimiento consumo de los hogares Vs. Crecimiento del ingreso

$1956-2002$

Fuente: Banco de la República
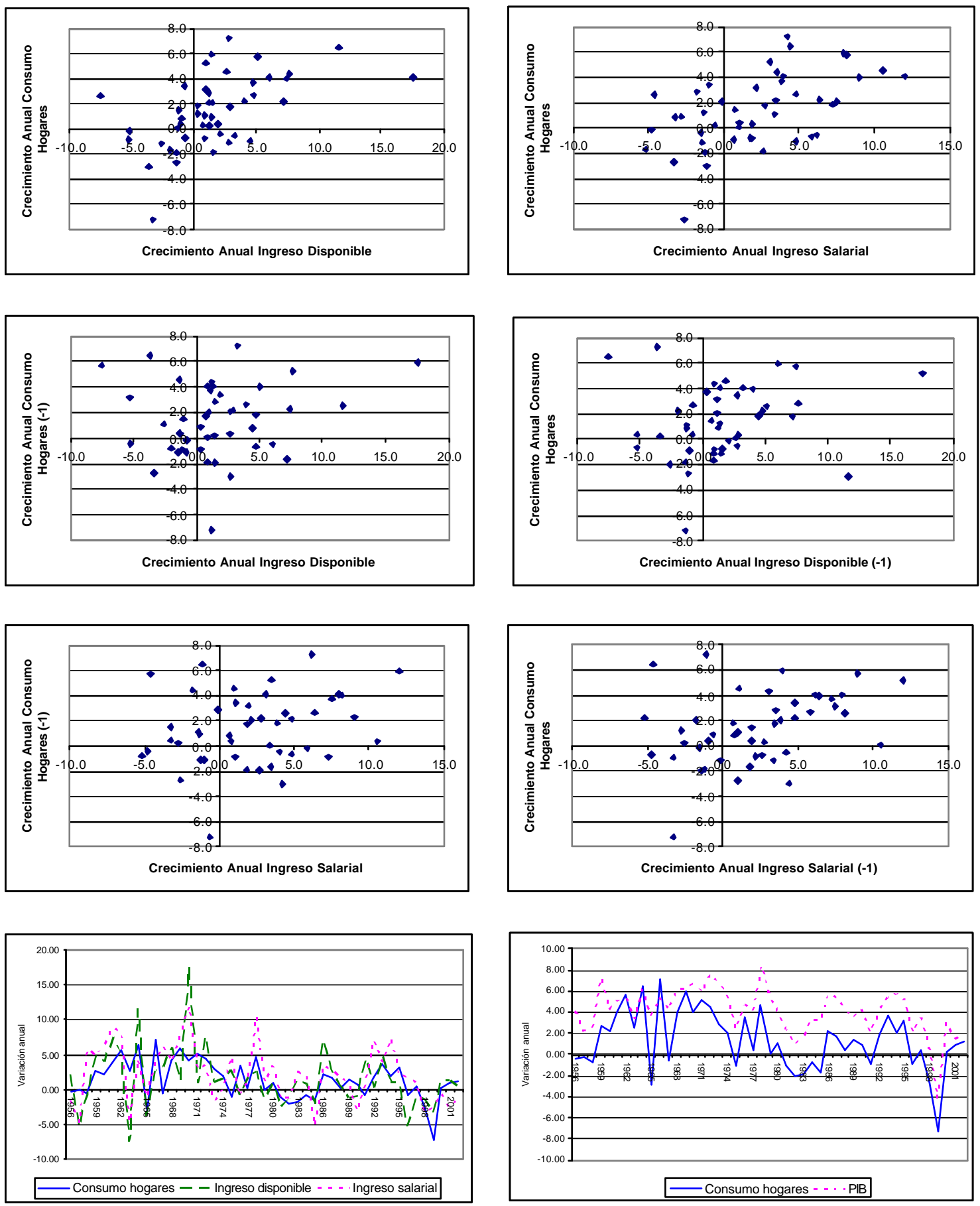
Anexo 3b

Crecimiento del consumo de los Hogares y del Stock de vivienda calculado según metodología de Harberger, 1956 -2002

Cálculos a partir de datos DANE-Banco de la República

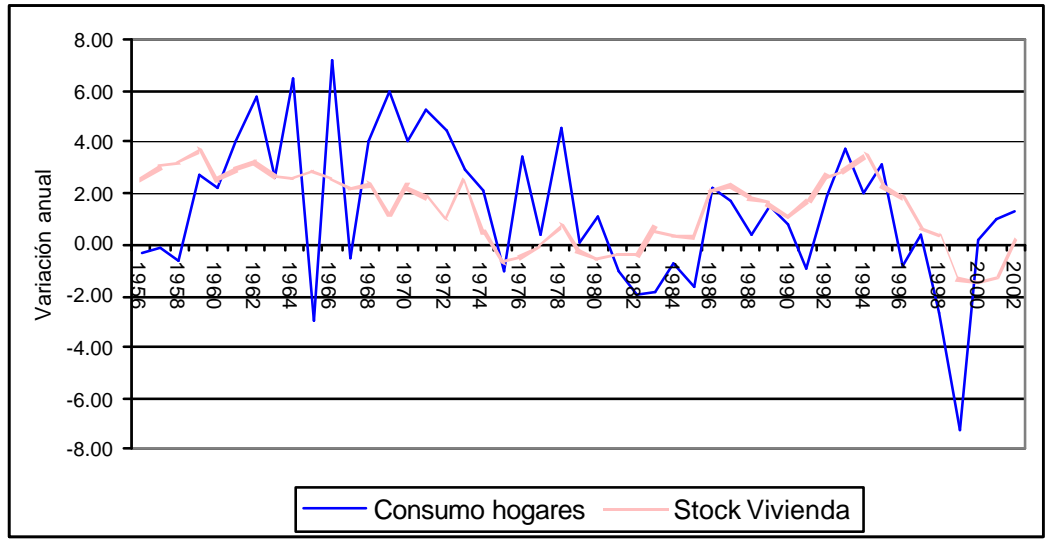

Anexo 3c

Estructura Demográfica y Consumo de los Hogares

$1956-2002$

Fuente: DNP, DANE y Banco de la República

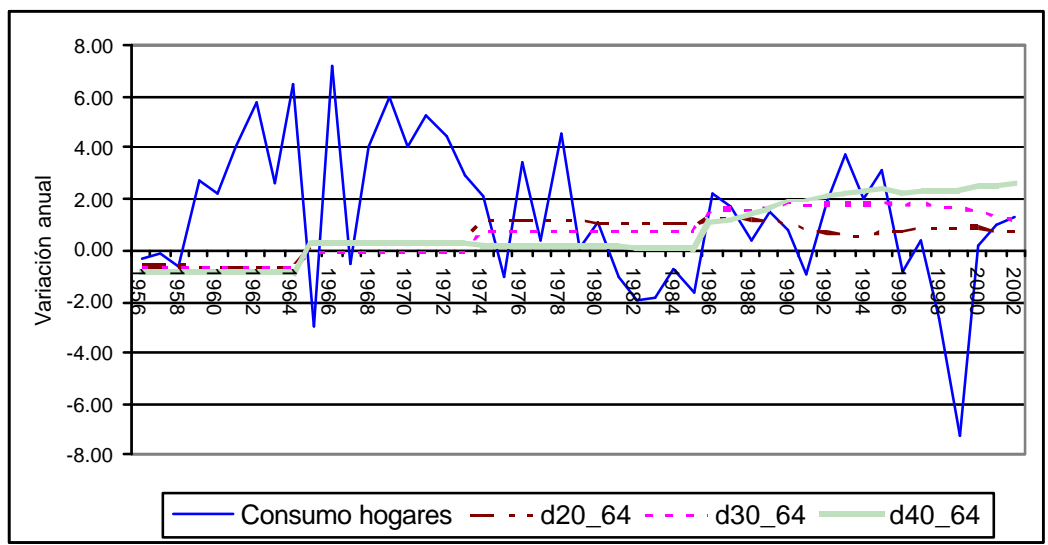


Anexo 3d

Restricciones Crediticias y Consumo de los Hogares

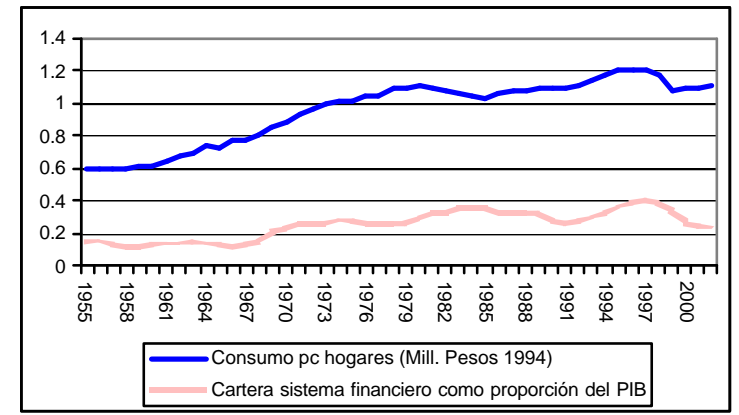

Fuente: DANE - Banco de la República

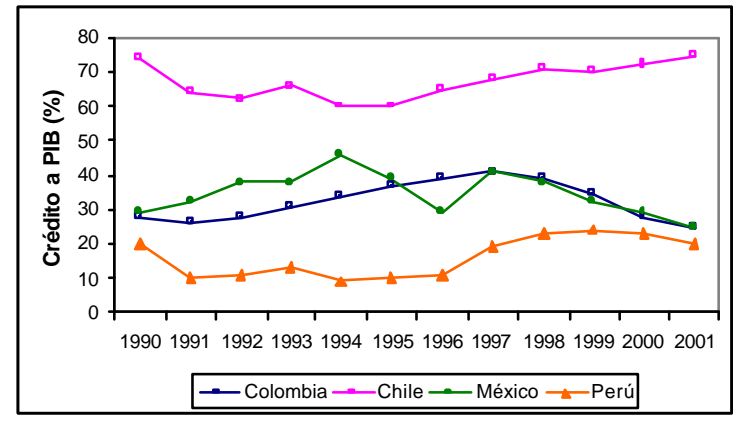

Fuente: Banco Mundial
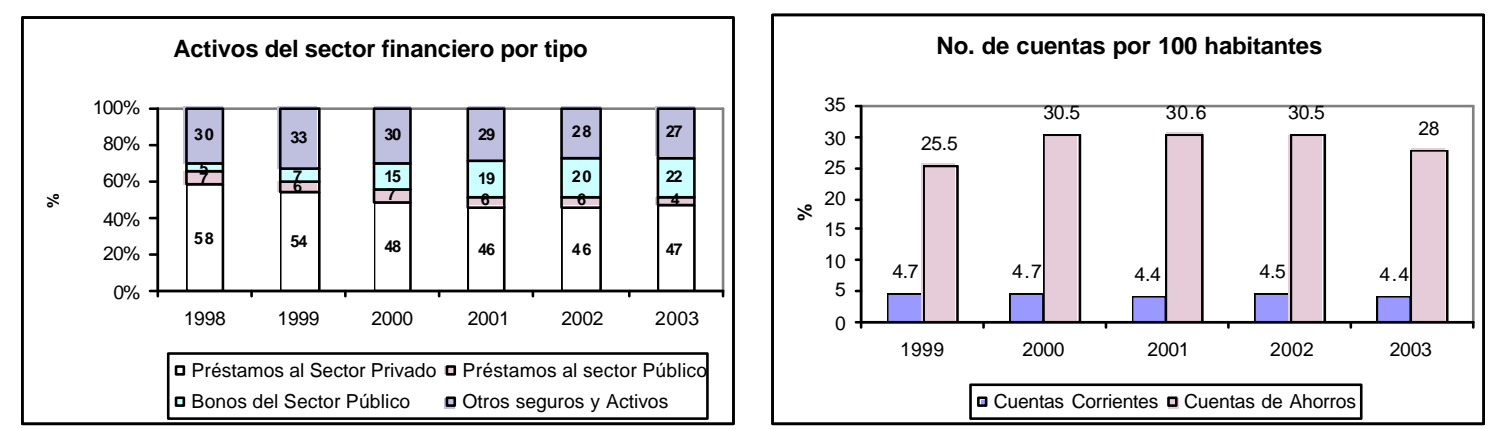

Fuente: Banco Mundial, Superintendencia Financiera, Asobancaria.

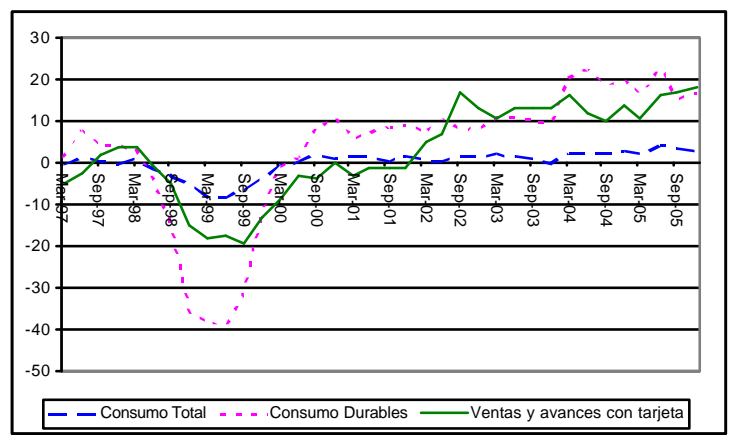

Fuente: DANE - Redeban Multicolor 


\section{Anexo 4 \\ Metodología de Estimación - Modelo Multivariado con Corrección de Errores (VEC)}

Los sistemas de información se amplían sucesivamente partiendo de un sencillo sistema bivariado que incluye el consumo de los hogares y el ingreso (en línea con la HIA) para posteriormente contemplar variables como dependencia demográfica, riqueza y acceso al crédito de acuerdo a los lineamientos teóricos expuestos anteriormente.

Se plantea en consecuencia la siguiente especificación $\Delta Y_{t}=\Gamma_{1} \Delta Y_{t-1}+\cdots+\Gamma_{k-1} \Delta Y_{t-k+1}+\Pi Y_{t-1}+\mu+\Psi D_{t}+\varepsilon_{t}$; siendo $Y_{t}$ el sistema de información compuesto por las variables sugeridas por determinada teoría, $D_{t}$ el conjunto de elementos determinísticos y $\Pi=\alpha \beta^{\prime}$ el producto entre, las velocidades de ajuste $\alpha$ y el respectivo vector de cointegración $\beta$.

Se trabajan con las series en logaritmo natural ${ }^{48}$ y se estima la existencia de la relación de largo plazo de uno a seis rezagos bajo los modelos: (i) drift (D), el cual incluye una tendencia lineal determinística en los niveles de las variables y (ii) cidrift (C) el cual reconoce la existencia de tendencia lineal en el vector de cointegración. Se sigue en consecuencia la selección de componentes determinísticas propuesta por Johansen (1994).

Una vez que se encuentra existencia de cointegración ${ }^{49}$ y el comportamiento de los residuales se juzga como adecuado al ser ruido blanco y normal multivariado (Lütkepohl (1993)) los modelos se someten a pruebas de exogeneidad débil y exclusión del vector de cointegración. Es de señalar que, dado el propósito del trabajo se requiere que el consumo no presente evidencia de exogeneidad débil y que ninguna de las variables del sistema este excluida del vector de cointegración.

\footnotetext{
${ }^{48}$ Con excepción de la tasa de interés.

${ }^{49}$ Es de señalar que, en el análisis de cointegración se consideran variables "dummies" que recogen valores atípicos cuando así se requiera. Además en la determinación del rango por el estadístico de la traza se tiene en cuenta qué de acuerdo a Cheung y Lai (1993) dicha prueba muestra más asimetría y exceso de curtosis que el de máximo valor propio (en los residuos) por lo cual se requiere una corrección previa por tamaño de muestra.
} 
En caso de no encontrar evidencia para rechazar h hipótesis de exogeneidad débil de las variables distintas al consumo, se estiman las elasticidades de largo plazo considerando el sistema bajo restricciones de exogeneidad débil. Ello consiste fundamentalmente en imponer un cero sobre las velocidades de ajuste $\alpha_{i}$ de manera que el desequilibrio de largo plazo no afecta a las ecuaciones de corto plazo de las variables que se estiman exógenas débiles $^{50}$.

Para obtener las elasticidades es importante observar que por lo general en la literatura empírica se han asociado las mismas al vector de cointegración. Sin embargo y como señala Misas et al (2001), citando a Johansen, esta interpretación es controversial pues ignora la dinámica de largo y corto plazo. Se sugieren entonces formas alternativas para obtener dichas elasticidades como el análisis de impulso respuesta o la matriz C fruto de la representación $\mathrm{MA}^{51}$ del sistema:

$$
\begin{gathered}
Y_{t}=C \sum_{i=1}^{t}\left(\varepsilon_{i}+\Psi D_{i}\right)+C(L)\left(\varepsilon_{t}+\Psi D_{t}\right)+P_{\beta_{\perp}} Y_{0} \\
\text { donde } C=\beta_{\perp}\left(\alpha^{\prime}{ }_{\perp} \Gamma \beta_{\perp}\right)^{-1} \alpha^{\prime \prime}{ }_{\perp} \text { y } P_{\beta_{\perp}}=\beta_{\perp}\left(\beta^{\prime}{ }_{\perp} \beta_{\perp}\right)^{-1} \beta^{\prime}
\end{gathered}
$$

De tal forma que un choque aleatorio en el momento $t=1$, sobre una de las ecuaciones del sistema, tiene un efecto de corto plazo a través de $C(L) \varepsilon_{t}$, el cual desaparece en el tiempo, y un efecto de largo plazo en la matriz C a través de la columna correspondiente a la ecuación bajo el choque.

\footnotetext{
${ }^{50}$ Desde luego se observa que los residuales mantengan un comportamiento adecuado tras la imposición de las restricciones.

${ }^{51}$ Moving average.
} 


\section{Anexo 5 \\ Tabla resumen de las Estimaciones ${ }^{52}$}

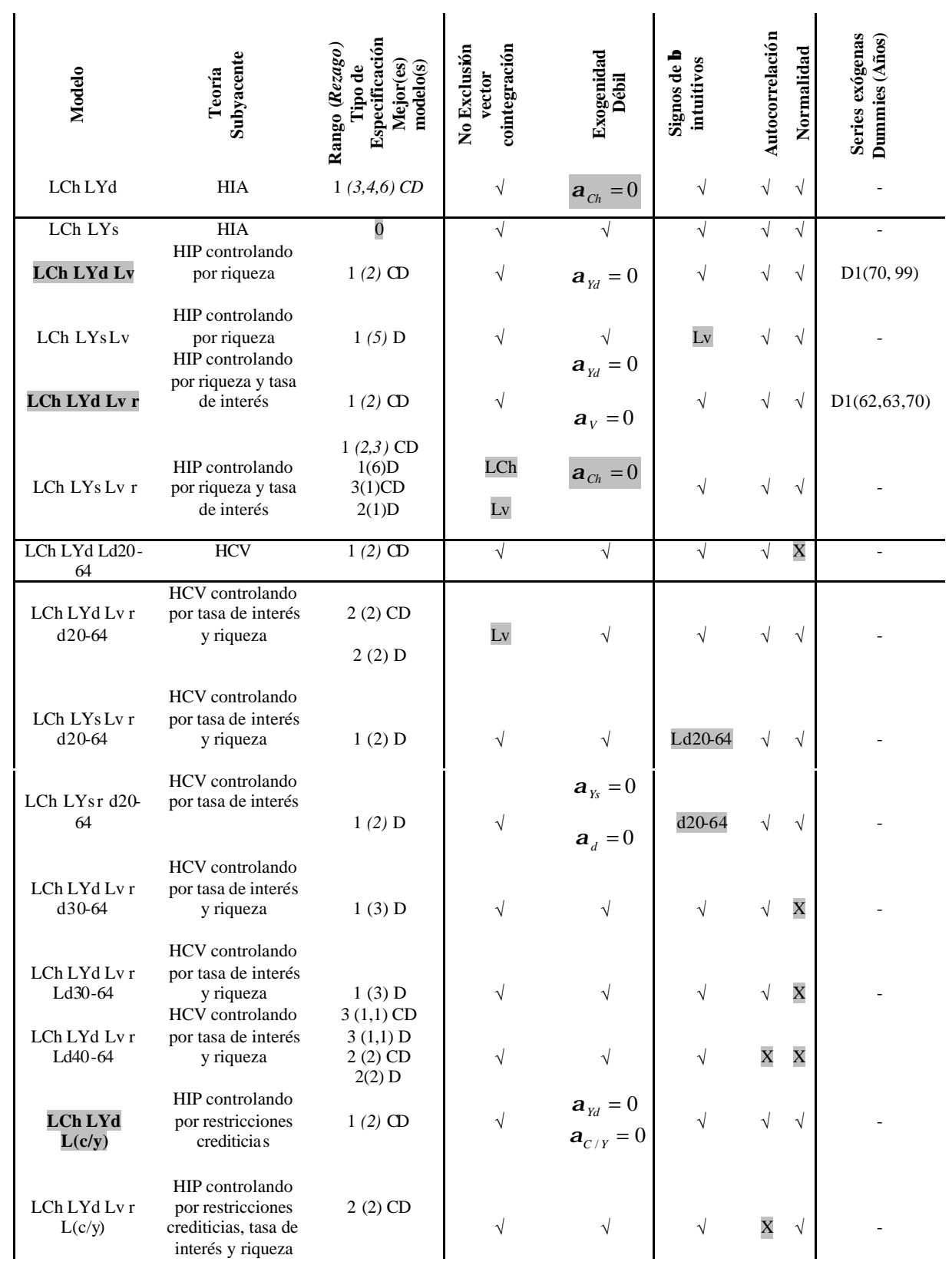

\section{Convenciones:}

L:Logaritmo natural, Ch: Consumo de los Hogares, Yd: Ingreso Disponible, Ys: Ingresos salariales, v: Stock de vivienda (riqueza), d $x-y$ : Población entre $x$ y $y$ años en relación a la población total, c/y: Cartera total del sistema financiero como proporción del PIB, r: Tasa de Interés.

\footnotetext{
${ }^{52}$ Los modelos sombreados se refieren a aquellos que cumplen satisfactoriamente con los criterios del análisis de cointegra ción. Para aquellos sistemas que fallan en algún aspecto se resalta el problema.
} 


\section{Anexo 6 \\ Especificaciones que satisfacen los criterios del Análisis de Cointegración}

\begin{tabular}{|c|c|c|c|c|c|c|c|}
\hline \multirow[b]{2}{*}{ Sistema } & \multirow[b]{2}{*}{$\begin{array}{l}\text { Modelo } \\
\text { (Rezago) }\end{array}$} & \multirow[b]{2}{*}{$\begin{array}{c}\alpha_{x}=0 \\
*\end{array}$} & \multirow{2}{*}{$\begin{array}{c}\text { Vector de } \\
\text { Cointegración } \\
\text { (Variables, } \\
\text { Tendencia)** }\end{array}$} & \multirow[b]{2}{*}{ Normalidad } & \multicolumn{3}{|c|}{ Autocorrelación } \\
\hline & & & & & LB & $\operatorname{LM}(1)$ & LM(4) \\
\hline LCh, LYd, Lv * & CD (2) & $\alpha_{Y d}$ & $\left(\begin{array}{llll}1 & -0.814 & -1.06 & 0.013\end{array}\right)$ & 0.47 & 0.05 & 0.14 & 0.18 \\
\hline LCh, LYd, Lv, r $\diamond$ & CD (2) & $\alpha_{Y d}$ & $\begin{array}{c}(1-0.792-0.616-0.608 \\
0.008)\end{array}$ & 0.18 & 0.63 & 0.12 & 0.25 \\
\hline LCh, LYd,LC_Y & $\mathrm{CD}(2)$ & $\begin{array}{c}\alpha_{Y d} \\
\alpha_{C_{-} Y}\end{array}$ & $\left(\begin{array}{llll}1 & -1.234 & 0.204 & 0.003\end{array}\right)$ & 0.03 & 0.15 & 0.22 & 0.11 \\
\hline
\end{tabular}

* Restricción de exogeneidad débil sobre la variable $\mathrm{x}$

** Recuerde que los signos por efectos de la estimación están con el signo contrario

* La variable de intervención considerada es dum1 (1970, 1999)

$\diamond \quad$ La variable de intervención considerada es dum1 $(1962,1963,1970)$

\section{Elasticidades}

Como se mencionó anteriormente es preferible interpretar las elasticidades a partir de la matriz Co adicionalmente hacer su lectura basándose en la función impulso respuesta. Bajo este último enfoque se obtuvo la respuesta en el logaritmo natural del consumo ante un choque de una unidad en la variable relacionada.

En el siguiente cuadro los resultados que se observan bajo la columna titulada Impulso Respuesta se refieren al valor que toma el logaritmo del consumo una vez se estabiliza la respuesta tras el choque. El impulso respuesta en diferencias hace alusión a la suma de las primeras diferencias del impulso respuesta. La obtención de las mismas bajo esta última aproximación se sustenta en el hecho de que en el caso continuo, la primera derivada del logaritmo puede aproximarse a la variación porcentual.

\begin{tabular}{c|c|c|c|}
\hline Sistema LCh : & Matriz C & $\begin{array}{c}\text { Impulso Respuesta en } \\
\text { Diferencias }\end{array}$ & Impulso Respuesta \\
\hline LYd Lv & $(0.106,3.822)$ & $(0.39,3.487)$ & $(0.78,4.26)$ \\
\hline LYd Lv r & $(0.533,2.964,-0.11)$ & $(0.5362 .9059-0.158)$ & $(0.84,3.64,-0.047)$ \\
\hline LYd LC_Y & $(1,122-0.163)$ & $(0.7528-0.1943)$ & $(1.088-0.154)$ \\
\hline
\end{tabular}


Medidas de Error de Pronóstico

$$
\begin{aligned}
& \mathbf{U}-\mathbf{T H E I L}=\sqrt{\frac{\sum_{t=1}^{n} \hat{\varepsilon}_{t}^{2}}{\left(y_{t}-y_{t-1}\right)}} \\
& \mathbf{R M P E}=\sqrt{\frac{1}{n} \sum_{t=1}^{n}\left(y_{t}-\hat{y}_{t}\right)^{2}} \\
& \mathbf{R M S P E}=\sqrt{\frac{1}{n} \sum_{t=1}^{n}\left[\frac{y_{t}-\hat{y}_{t}}{y_{t}}\right]^{2}} \\
& \text { MAE }=\frac{1}{n} \sum_{t=1}^{n}\left|y_{t}-\hat{y}_{t}\right| \\
& \text { MAPE }=\frac{1}{n} \sum_{t=1}^{n}\left|\frac{y_{t}-\hat{y}_{t} \mid}{y_{t}}\right|
\end{aligned}
$$


Anexo 8

Evaluación de Pronóstico de los Modelos Estimados

U de Theil Promedio

\begin{tabular}{|c|c|cc|c|}
\hline \multicolumn{5}{|c|}{ U de Theil Promedio Sistema \{lch, lyd, lv,r\} } \\
Evaluación Fuera de Muestra 1997-2001 \\
\hline Un año & Dos años & Tres años & Cuatro años & Cinco años \\
\hline 3.6 & 2.88 & 4.25 & 3.91 & 4.7 \\
\hline
\end{tabular}

\begin{tabular}{|c|c|cc|c|}
\hline \multicolumn{5}{|c|}{ U de Theil Promedio Sistema \{lch, lyd, lv, r\} } \\
Evaluación Fuera de Muestra 1997-2001 \\
\hline Un año & Dos años & Tres años & Cuatro años & Cinco años \\
\hline 2.47 & 1.91 & 2.63 & 1.94 & 2.63 \\
\hline
\end{tabular}

\begin{tabular}{|c|c|cc|c|}
\hline \multicolumn{5}{|c|}{ U de Theil Promedio Sistema $\{$ Ich, lyd, lc_y $\}$} \\
Evaluación Fuera de Muestra 1997-2001 \\
\hline Un año & Dos años & Tres años & Cuatro años & Cinco años \\
\hline 3.34 & 2.78 & 2.54 & $\underline{0.88}$ & 1.04 \\
\hline
\end{tabular}

U de Theil por años. Sistema con proxy de acceso al crédito.

\begin{tabular}{|c|c|c|c|c|c|c|}
\hline \multicolumn{7}{|c|}{ U de Theil Ich, lyd, Ic_y } \\
\hline Horizonte & $\mathbf{1 9 9 7}$ & $\mathbf{1 9 9 8}$ & $\mathbf{1 9 9 9}$ & $\mathbf{2 0 0 0}$ & $\mathbf{2 0 0 1}$ & Promedio \\
\hline Un año & $\mathbf{0 . 0 1}$ & $\mathbf{0 . 6 3}$ & 11.65 & 4.06 & $\mathbf{0 . 3 7}$ & 3.34 \\
Dos años & $\mathbf{0 . 7 3}$ & $\mathbf{0 . 7 4}$ & 6.13 & 3.54 &. & 2.78 \\
Tres años & $\mathbf{0 . 9 5}$ & $\mathbf{0 . 7 5}$ & 5.94 &. &. & 2.54 \\
Cuatro años & 1.02 & $\mathbf{0 . 7 4}$ &. &. &. & 0.88 \\
Cinco Años & 1.04 &. &. &. &. & 1.04 \\
\hline
\end{tabular}

Otras Medidas de Bondad del Pronóstico

\begin{tabular}{|c|c|c|c|c|}
\hline \multicolumn{5}{|c|}{ Ich, lyd, lc y } \\
\hline Rolling & RMSE & RMSPE & MAE & MAPE \\
\hline Un año & 0.03 & 0.02 & 0.03 & 0.02 \\
Dos años & 0.04 & 0.04 & 0.04 & 0.04 \\
Tres años & 0.05 & 0.04 & 0.04 & 0.04 \\
Cuatro años & 0.04 & 0.04 & 0.03 & 0.03 \\
Cinco Años & 0.04 & 0.04 & 0.04 & 0.03 \\
\hline
\end{tabular}




\begin{tabular}{|c|c|c|c|c|}
\hline \multicolumn{5}{|c|}{ Ich, lyd, Iv } \\
\hline Rolling & RMSE & RMSPE & MAE & MAPE \\
\hline Un año & 0.05 & 0.05 & 0.05 & 0.05 \\
Dos años & 0.08 & 0.07 & 0.07 & 0.07 \\
Tres años & 0.13 & 0.11 & 0.12 & 0.11 \\
Cuatro años & 0.17 & 0.16 & 0.16 & 0.15 \\
Cinco Años & 0.19 & 0.18 & 0.18 & 0.16 \\
\hline
\end{tabular}

\begin{tabular}{|c|c|c|c|c|}
\hline \multicolumn{5}{|c|}{ Ich, lyd, Iv, r } \\
\hline Rolling & RMSE & RMSPE & MAE & MAPE \\
\hline Un año & 0.03 & 0.03 & 0.03 & 0.03 \\
Dos años & 0.05 & 0.04 & 0.04 & 0.04 \\
Tres años & 0.07 & 0.06 & 0.07 & 0.06 \\
Cuatro años & 0.09 & 0.08 & 0.08 & 0.08 \\
Cinco Años & 0.11 & 0.10 & 0.10 & 0.09 \\
\hline
\end{tabular}


Anexo 9

Estabilidad del Vector de Cointegración

Sistema de información $\{$ lch, lyd, lv\}

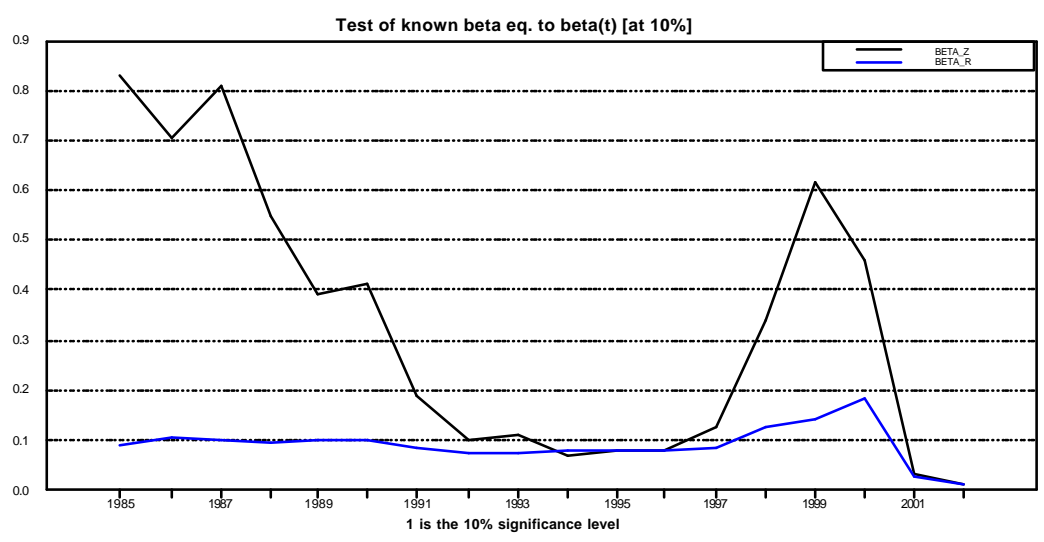

Sistema de información $\{$ lch, lyd, lv,r\}

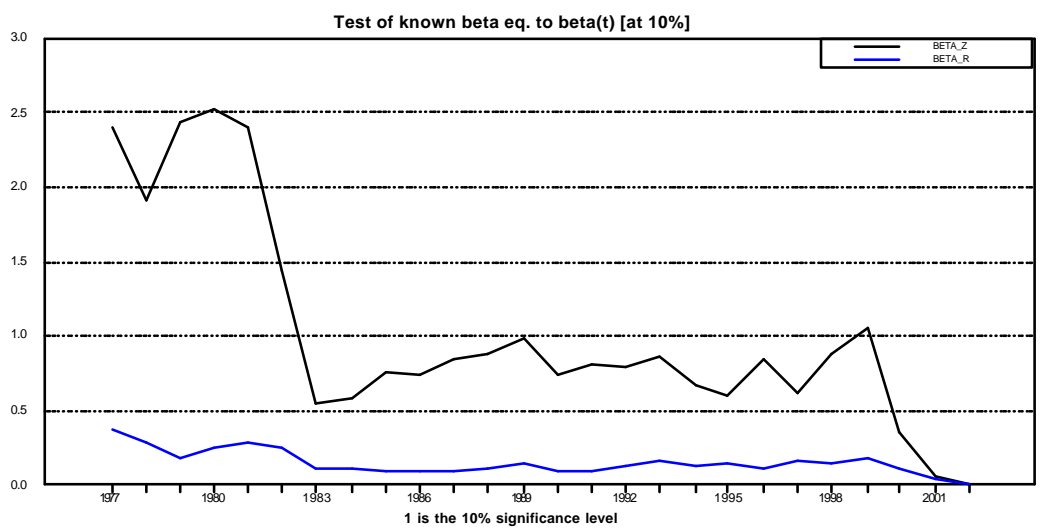

Sistema de información $\{$ Ich, lyd, lc_y\}

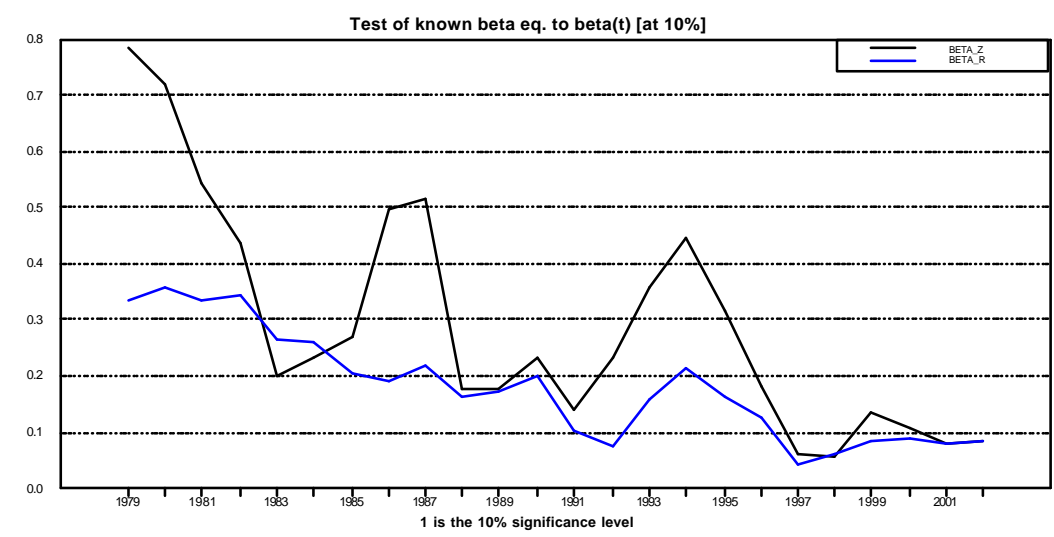

